\title{
Relative normalizers of automorphism groups, infravacua and the problem of velocity superselection in QED
}

\author{
Daniela Cadamuro* and Wojciech Dybalski ${ }^{\dagger}$ \\ Zentrum Mathematik, Technische Universität München, \\ D-85747 Garching, Germany
}

\begin{abstract}
We advance superselection theory of pure states on a $C^{*}$-algebra $\mathfrak{A}$ outside of the conventional (DHR) setting. First, we canonically define conjugate and second conjugate classes of such states with respect to a given reference state $\omega_{\text {vac }}$ and background $a \in \operatorname{Aut}(\mathfrak{A})$. Next, for some subgroups $R \varsubsetneqq S \varsubsetneqq G \subset \operatorname{Aut}(\mathfrak{A})$ we study the family $\left\{\omega_{\text {vac }} \circ s \mid s \in S\right\}$ of infrared singular states whose superselection sectors may be disjoint for different $s$. We show that their conjugate and second conjugate classes always coincide provided that $R$ leaves the sector of $\omega_{\text {vac }}$ invariant and $a$ belongs to the relative normalizer $N_{G}(R, S):=\left\{g \in G \mid g \cdot S \cdot g^{-1} \subset R\right\}$. We study the basic properties of this apparently new group theoretic concept and show that the Kraus-PolleyReents infravacuum automorphisms belong to the relative normalizers of the automorphism group of a suitable CCR algebra. Following up on this observation we show that the problem of velocity superselection in non-relativistic QED disappears at the level of conjugate and second conjugate classes, if they are computed with respect to an infravacuum background. We also demonstrate that for more regular backgrounds such merging effect does not occur.
\end{abstract}

\section{Introduction}

The infrared problem is a maze of difficulties in spectral, scattering and superselection theory of quantum systems which can be traced back to the presence of massless particles and long-range forces. This topic enjoys currently some revival in the highenergy physics community triggered by a proposal of Hawking, Perry and Strominger to solve the black hole information paradox using the infrared degrees of freedom of the gravitational field [HPS16]. This proposal relies on an implicit assumption that these infrared degrees of freedom can be measured, in particular have sufficiently mild

*E-mail: daniela.cadamuro@itp.uni-leipzig.de

${ }^{\dagger}$ E-mail: dybalski@ma.tum.de 
fluctuations. In the case of QED this assumption (cf. [Bu86, formula (2b)]) lies at the heart of the infrared problem. Its consequences include several closely related pathologies which can collectively be called the infraparticle problem: superselection of the electron's velocity [Fr73, CF07, CFP09, KM14, DT12], the absence of the sharp mass of the electron [Bu86, HH08], and infrared divergencies in the Dyson scattering matrix [We]. In view of the above, a natural approach to cure the infrared problems is to immerse the system in a low-energetic but highly fluctuating radiation which blurs the infrared degrees of freedom. A concrete example of such an infravacuum was given by Kraus, Polley and Reents already four decades ago in the context of the quantized electromagnetic field coupled to an external current [Re74, KPR77, Kr82]. The initial success of the approach was a well-defined Dyson scattering matrix in this exactly solvable situation. However, the problem of velocity superselection and sharp mass of the electron were not treated in these works, mostly due to the absence (back then) of mathematically tractable, translation-invariant models of QED and limited understanding of superselection theory in the presence of long-range forces. As the intervening decades witnessed progress in mathematical control of such systems in the infrared regime (see, e.g., [BFS98, BCFFS13, Pi03, Pi05, CF07, CFP07, CFP09, HH08, Hi00, KM14, LMS07, DP13, DP18]) and substantial advances on the side of general superselection theory [Bu82,BR14] there is now every reason to re-initiate the infravacuum program.

In the present paper we point out that the infravacua exemplify a general grouptheoretic concept which apparently escaped attention so far: Let $R \varsubsetneqq S \varsubsetneqq G$ be subgroups of a group $G$ and let us call

$$
N_{G}(R, S):=\left\{g \in G \mid g \cdot S \cdot g^{-1} \subset R\right\}
$$

the relative normalizer of the pair of subgroups $(R, S)$. Due to the tension between the inclusion $R \varsubsetneqq S$ and the opposite inclusion $g \cdot S \cdot g^{-1} \subset R$ in the definition of $N_{G}(R, S)$, one can immediately exclude non-empty relative normalizers in many situations. In particular, relative normalizers are empty for any subgroups of an abelian group $G$. The same is true for finite groups (abelian or not), since elements of the relative normalizer would provide bijections between sets of different cardinality. Similarly, closed, connected subgroups of finite-dimensional Lie groups have empty relative normalizers, as their elements would provide continuous bijections between sets of different dimension (cf. [Sch16, Proposition 5.1, Theorem 5.6]). Finding nontrivial examples of relative normalizers is also not difficult, for example in the context of infinite discrete groups (see Subsection 2.1). However, our main interest in this paper is in a certain concrete class of relative normalizers, which is relevant for infrared problems: Let $\mathcal{L}$ be the photon single particle space with a suitable IR regularisation. We show that the inhomogeneous symplectic group $\operatorname{ISp}(\mathcal{L})$ admits relative normalizers which contain the Kraus-Polley-Reents symplectic maps $T$ (cf. Subsection 3.1, Theorem 4.3 and Definition 5.1). These relative normalizers can be lifted to the group of automorphisms $\operatorname{Aut}(\mathfrak{A})$ of the corresponding CCR algebra $\mathfrak{A}$. Indeed, with the help of the automorphism $\alpha$ which maps elements of $\operatorname{ISp}(\mathcal{L})$ into the corresponding Bogoliubov transformations in $\operatorname{Aut}(\mathfrak{A})$ we show that $\alpha_{T}$ belongs to the relative normalizer in any subgroup $G$ of $\operatorname{Aut}(\mathfrak{A})$ containing $\alpha_{\operatorname{ISp}(\mathcal{L})}$. In this context $R$ and $S$ are the subgroups of automorphisms $\alpha_{\mathcal{L}_{\mathrm{R}}^{*}}$ and $\alpha_{\mathcal{L}_{\mathrm{S}}^{*}}$ of $G$, where $\mathcal{L}_{\mathrm{R}}^{*}$ is a subspace of the 
single photon space without IR regularisation and $\mathcal{L}_{\mathrm{S}}^{*}$ is the sum of $\mathcal{L}_{\mathrm{R}}^{*}$ with the set of infrared singular dressing transformations of the bare electron for different momenta.

Our interest in relative normalizers derives from their relevance for superselection theory of the $C^{*}$-algebra $\mathfrak{A}$. Let $P_{\mathfrak{A}}$ be the set of pure states and $X:=P_{\mathfrak{A}} / \operatorname{In}(\mathfrak{A})$ the set of sectors. The latter are the orbits of states under the action of inner automorphisms of $\mathfrak{A}$ (cf. [BR14, Definition 4.1]) and for a state $\omega \in P_{\mathfrak{A}}$ such orbit will be denoted by $[\omega]_{\operatorname{In}(\mathfrak{A})}$. Now we consider the natural right action of a subgroup $G \subset \operatorname{Aut}(\mathfrak{A})$ on $X$, fix a reference ('vacuum') sector $x_{0}=\left[\omega_{\text {vac }}\right]_{\operatorname{In}(\mathfrak{A})}$ and a 'background automorphism' $a \in G$ corresponding to background radiation. With this data, for any sector $x \in X$ we define the conjugate class in a way which is suggested by the DHR superselection theory of simple charges [Ha]:

$$
\overline{[x]}^{a}:=\left\{x_{0} \cdot a \cdot g^{-1} \mid g \in G_{x, x_{0}}^{a}\right\}, \text { where } G_{x, x_{0}}^{a}:=\left\{g \in G \mid x=x_{0} \cdot a \cdot g\right\} .
$$

By iterating this procedure, we also define the second conjugate class $\overline{\overline{[x]}}^{a}$. Now let $R \varsubsetneqq S \varsubsetneqq G$ be subgroups as above and let us consider the family $\left\{x_{0} \cdot s \mid s \in S\right\}$ of singular sectors. Our main general result is that

$$
{\overline{\left[x_{0} \cdot s_{1}\right]}}^{a}={\overline{\left[x_{0} \cdot s_{2}\right]}}^{a} \text { and }{\overline{\overline{\left[x_{0} \cdot s_{1}\right]}}}^{a}={\overline{\overline{\left[x_{0} \cdot s_{2}\right]}}}^{a}
$$

hold for all $s_{1}, s_{2} \in S$, provided that $x_{0} \cdot r=x_{0}$ for all $r \in R$ and $a \in N_{G}(R, S)$. These equalities are non-trivial as long as $x_{0} \cdot s_{1} \neq x_{0} \cdot s_{2}$ for some $s_{1} \neq s_{2}$ and we give such examples below. We also demonstrate that the identities (1.3) may fail, if the background $a$ is not in $N_{G}(R, S)$. We stress that the above considerations are purely group-theoretical, and we referred to $\operatorname{Aut}(\mathfrak{A})$ only for concreteness and motivation.

If a sector $x \in X$ belongs to the $G$-orbit of $x_{0}$, it is easy to see that $x \in \overline{\overline{[x]}}^{a}$. More than that, if $a \in N_{G}(R, S)$ then also $x \cdot s \in \overline{\overline{[x]}}^{a}$ for all $s \in S$ (cf. Theorem 2.9 (c)). In the context of the physical example below, the second conjugate class with respect to an infravacuum background absorbs a multitude of distinct sectors $x \cdot s, s \in S$, which differ only by physically irrelevant soft-photon clouds. Thus the second conjugate class appears to be a natural generalisation of the concept of a sector for theories with infrared problems. It is similar - in intention - to the charge classes introduced in [Bu82,BR14], but it does not rely on locality. Also, in contrast to earlier discussions of superselection theory with respect to infravacuum backgrounds [Bu82, Ku, Ku98.1], we do not use any variant of the DHR criterion. This facilitates applications of our general results to non-relativistic, interacting models of QED, as we now summarize.

The Hilbert space of the model we consider is $\mathcal{H}=L^{2}\left(\mathbb{R}^{3}\right) \otimes \mathcal{F}_{\mathrm{ph}}$, where $L^{2}\left(\mathbb{R}^{3}\right)$ carries the electron degrees of freedom and $\mathcal{F}_{\mathrm{ph}}$ is the Fock space of the physical photon states. The Hamiltonian has the standard form [Sp]

$$
H:=\frac{1}{2}\left(-i \nabla_{\boldsymbol{x}}+\tilde{\alpha}^{1 / 2} \boldsymbol{A}(\boldsymbol{x})\right)^{2}+H_{\mathrm{ph}}
$$

where $\tilde{\alpha}>0$ is the coupling constant, $\boldsymbol{x}$ is the position of the electron, $\boldsymbol{A}$ is the electromagnetic potential in the Coulomb gauge with fixed ultraviolet regularization and $H_{\mathrm{ph}}$ is the free photon Hamiltonian. This Hamiltonian is translation-invariant, that is, it commutes with the total momentum operators $\boldsymbol{P}=-i \nabla_{\boldsymbol{x}}+\boldsymbol{P}_{\mathrm{ph}}$, where 
$\boldsymbol{P}_{\mathrm{ph}}$ is the free-photon momentum. Consequently, we can decompose it into the fiber Hamiltonians $H_{P}$ at fixed momentum

$$
H=\Pi^{*}\left(\int^{\oplus} H_{\boldsymbol{P}} d^{3} \boldsymbol{P}\right) \Pi,
$$

where $\Pi$ is a certain unitary identification. The Hamiltonians $H_{\boldsymbol{P}}$, precisely defined in Section 4.1 below, are self-adjoint operators acting on the fiber Fock space which we denote by $\mathcal{F}$. It is one manifestation of the infraparticle problem that $H_{\boldsymbol{P}}$ do not have ground states for $\boldsymbol{P} \neq 0$, at least for small $\tilde{\alpha}$ and for $\boldsymbol{P}$ in some ball $\mathcal{S}$ around zero [HH08, CFP09]. By introducing an infrared cut-off $\sigma>0$ in the interaction term in (1.4), we obtain the Hamiltonian $H_{\sigma}$ and the corresponding fiber Hamiltonians $H_{\boldsymbol{P}, \sigma}$, which do have the (normalized) ground states $\Psi_{\boldsymbol{P}, \sigma}$ in the same region of parameters $\tilde{\alpha}, \boldsymbol{P}$. Although these vectors tend weakly to zero as $\sigma \rightarrow 0$ [CFP09], one obtains well-defined states on a certain CCR algebra $\mathfrak{A}^{1}$

$$
\omega_{\boldsymbol{P}}(A):=\lim _{\sigma \rightarrow 0}\left\langle\Psi_{\boldsymbol{P}, \sigma}, \pi_{\mathrm{vac}}(A) \Psi_{\boldsymbol{P}, \sigma}\right\rangle, \quad A \in \mathfrak{A},
$$

where $\pi_{\text {vac }}$ is the Fock vacuum representation. These states can be interpreted as plane-wave configurations of the electron moving with momentum $\boldsymbol{P}$. It is well known that in (1.4), and in similar models of non-relativistic QED, the GNS representations $\pi_{\boldsymbol{P}}$ of the states $\omega_{\boldsymbol{P}}$ are disjoint for different values of $\boldsymbol{P} \in \mathcal{S}[\mathrm{Fr} 73, \mathrm{CF} 07, \mathrm{CFP} 09$, KM14]. This mathematical formulation of velocity superselection was first introduced by Fröhlich in [Fr73].

Given the $C^{*}$-algebra $\mathfrak{A}$ and the family of distinct sectors $\left[\omega_{\boldsymbol{P}}\right]_{\operatorname{In}(\mathfrak{A})} \neq\left[\omega_{\boldsymbol{P}^{\prime}}\right]_{\operatorname{In}(\mathfrak{A})}$ for $\boldsymbol{P} \neq \boldsymbol{P}^{\prime}$ we can compute their conjugate and second conjugate classes. We choose the Fock vacuum $\left[\omega_{\text {vac }}\right]_{\operatorname{In}(\mathfrak{A})}$ as a reference sector and a Kraus-Polley-Reents infravacuum automorphism $\alpha_{T}$ as a background. By exhibiting a concrete relative normalizer including $\alpha_{T}$, we obtain from (1.3) that

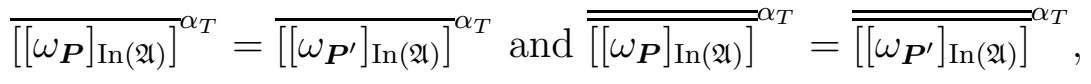

for all $\boldsymbol{P}, \boldsymbol{P}^{\prime} \in \mathcal{S}$. Thus velocity superselection disappears at the level of conjugate and second conjugate classes. Furthermore, if $\alpha_{T}$ in (1.7) is replaced with some regular background (e.g., the identity automorphism), the velocity superselection persists, at least for $\boldsymbol{P}=0, \boldsymbol{P}^{\prime} \neq 0$. These results, stated precisely in Theorem 4.5 below, illustrate the utility of our general theory in a concrete model.

Our paper is organized as follows: In Section 2 we introduce the concepts of relative normalizers and (second) conjugate classes, and prove their general properties. We also explain the relevance of these group-theoretic considerations to superselection theory of $C^{*}$-algebras. Section 3 concerns relative normalizers in the inhomogeneous symplectic group and in the automorphism group of the corresponding CCR algebra. These results are applied to the problem of velocity superselection in non-relativistic

\footnotetext{
${ }^{1}$ This CCR-algebra is defined in Subsections 3.2, 4.2 below and we give a proof of velocity superselection in our setting in Section 6. In earlier works [Fr73, CF07, CFP09, KM14] slightly different algebras are used. For example, in [CF07] $\mathfrak{A}=\overline{\bigcup_{\sigma>0} B\left(\mathcal{F}_{\sigma}\right)}\|\cdot\|$, where $\mathcal{F}_{\sigma} \subset \mathcal{F}$ is the subspace of functions vanishing in a ball of radius $\sigma$ around zero (in any variable).
} 
QED in Section 4. Section 5 covers the definition and basic properties of the KrausPolley-Reents infravacua and Section 6 gives a novel proof of velocity superselection.

Acknowledgements: We would like to thank Henning Bostelmann, Detlev Buchholz, Fabio Ciolli, Maximilian Duell, Simon Ruijsenaars and Yoh Tanimoto for discussions concerning the infravacuum representations. Thanks are also due to Jürg Fröhlich and Alessandro Pizzo for valuable remarks on non-relativistic QED. This work was supported by the DFG within the Emmy Noether grant DY107/2-1.

\section{Relative normalizers and conjugate classes}

\subsection{Relative normalizers}

Let $H \subset G$ be a subgroup of a group $G$. Recall that the normalizer of $H$ in $G$ is defined as

$$
N_{G}(H):=\left\{g \in G \mid g \cdot H \cdot g^{-1}=H\right\}
$$

and it is the largest subgroup of $G$ in which $H$ is normal. Also, we have the obvious relation:

$$
H \cdot N_{G}(H) \cdot H=N_{G}(H)
$$

We generalize this concept as follows:

Definition 2.1. Let $R \subset S \subset G$ be two subgroups of $G$. Then the relative normalizer of the pair $(R, S)$ in $G$ is defined as

$$
N_{G}(R, S):=\left\{g \in G \mid g \cdot S \cdot g^{-1} \subset R\right\} .
$$

Clearly, $N_{G}(R, S)$ is a semigroup, i.e., $N_{G}(R, S) \cdot N_{G}(R, S) \subset N_{G}(R, S)$, and similarly to $(2.2)$, we have

$$
R \cdot N_{G}(R, S) \cdot S=N_{G}(R, S) .
$$

If $N_{G}(R, S)$ is a group then $R=S$ and $N_{G}(S, S)=N_{G}(S)$. On the other hand, $R=S$ only implies $N_{G}(S, S) \supset N_{G}(S)$. The inclusion is proper and $N_{G}(S, S)$ fails to be a group exactly if $N_{G}(\tilde{R}, S)$ is non-empty for some subgroup $\tilde{R} \varsubsetneqq S$. Furthermore, for $S=G$ the condition $N_{G}(R, S) \neq \emptyset$ implies $R=G$ and $N_{G}(R, S)=G$. Leaving aside the case $N_{G}(S, S) \nsupseteq N_{G}(S)$, we will say that a relative normalizer $N_{G}(R, S)$ is non-trivial if $R \varsubsetneqq S \varsubsetneqq G$.

We note for future reference that relative normalizers behave naturally under group homomorphisms.

Lemma 2.2. Let $\varphi: G \rightarrow H$ be a group homomorphism and $R \subset S \subset G$ be subgroups of $G$. If $g \in N_{G}(R, S)$ then $\varphi(g) \in N_{\tilde{H}}(\varphi(R), \varphi(S))$, where $\tilde{H} \subset H$ is any subgroup containing $\varphi(G)$.

Proof. By assumption, $g \cdot s \cdot g^{-1} \in R$ for any $s \in S$. Hence $\varphi(g) \cdot \varphi(s) \cdot \varphi(g)^{-1}=$ $\varphi\left(g \cdot s \cdot g^{-1}\right) \subset \varphi(R)$.

The question of existence of non-trivial relative normalizers can readily be settled by the following example ${ }^{2}$ : Let $\mathfrak{S}_{\mathbb{Z}}$ be the permutation group of integers. Let $\mathfrak{S}_{\mathbb{N}}$, resp.

\footnotetext{
${ }^{2}$ We thank the anonymous referee for pointing out this example.
} 
$\mathfrak{S}_{\mathbb{N}_{0}}$ be the subgroups of $\mathfrak{S}_{\mathbb{Z}}$ consisting of permutations which act trivially on $\mathbb{Z} \backslash \mathbb{N}$, resp. $\mathbb{Z} \backslash \mathbb{N}_{0}$. Consider the inclusions $\mathfrak{S}_{\mathbb{N}} \subset \mathfrak{S}_{\mathbb{N}_{0}} \subset \mathfrak{S}_{\mathbb{Z}}$. Let $a \in \mathfrak{S}_{\mathbb{Z}}$ be a permutation which shifts each element by one to the right, i.e., $a(j)=j+1, j \in \mathbb{Z}$. Then it is easy to see that $a \in N_{\mathfrak{S}_{\mathbb{Z}}}\left(\mathfrak{S}_{\mathbb{N}}, \mathfrak{S}_{\mathbb{N}_{0}}\right)$. One can find similar examples in the context of diffeomorphism groups of intervals in $\mathbb{R}$ and unitary groups of Hilbert spaces.

\subsection{Group actions, orbits and conjugate classes}

Consider a group action of $G$ on a set $X$ which we denote $X \times G \ni(x, g) \mapsto x \cdot g$. (For future applications it is convenient to use the right action notation). For any $x \in X$ we write

$$
G_{x}:=\{g \in G \mid x \cdot g=x\}
$$

for the stabilizer subgroup of $x$. Furthermore, for any subgroup $H \subset G$ and $x \in X$ we denote the resulting orbit by

$$
[x]_{H}:=\{x \cdot h \mid h \in H\} .
$$

Motivated by the DHR superselection theory, we define for any $x \in X$ its conjugate and second conjugate class with respect to a certain reference point in $X$. This is a counterpart of the inverse operation in $G$.

Definition 2.3. Fix reference elements $x_{0} \in X$ and $a \in G$. For any $x \in X$ define the set $G_{x, x_{0}}^{a}:=\left\{g \in G \mid x=x_{0} \cdot a \cdot g\right\}$ and write

$$
\overline{[x]}^{a}:=\left\{x_{0} \cdot a \cdot g^{-1} \mid g \in G_{x, x_{0}}^{a}\right\}, \quad \overline{\overline{[x]}}^{a}:=\left\{x_{0} \cdot a \cdot\left(g^{\prime}\right)^{-1} \mid g^{\prime} \in G_{y, x_{0}}^{a}, y \in \overline{[x]}^{a}\right\} .
$$

We call $\overline{[x]}^{a}$, (resp. $\overline{\overline{[x]}}^{a}$ ) the conjugate (resp. second conjugate) class of $x$ with respect to $\left(x_{0}, a\right)$.

We note that $G_{x, x_{0}}^{a}$ is non-empty only if $x \in\left[x_{0}\right]_{G}$. Using this we obtain a simpler characterisation of conjugate classes in terms of orbits. In particular, it is clear from the following lemma that the conjugate classes do not change if $a$ is replaced with $g_{0} \cdot a$ for some $g_{0} \in G_{x_{0}}$.

Lemma 2.4. Let $x_{0}$, a be as in Definition 2.3 and suppose that $x=x_{0} \cdot g_{x}$ for some $g_{x} \in G$. Then

$$
\overline{[x]}^{a}=\left[x_{0} \cdot a \cdot g_{x}^{-1} \cdot a\right]_{a^{-1} \cdot G_{x_{0}} \cdot a}, \quad \overline{\overline{[x]}}^{a}=\left[x_{0} \cdot g_{x}\right]_{a^{-1} \cdot G_{x_{0}} \cdot a},
$$

where $G_{x_{0}}$ is the stabiliser group of $x_{0}$ and $a^{-1} \cdot G_{x_{0}} \cdot a=G_{x_{0} \cdot a}$ is the stabilizer group of $x_{0} \cdot a$.

Proof. Since $G_{x, x_{0}}^{a}:=\left\{g \in G \mid x_{0} \cdot g_{x}=x_{0} \cdot a \cdot g\right\}$, we have $G_{x, x_{0}}^{a}=a^{-1} \cdot G_{x_{0}} \cdot g_{x}$. Thus definition (2.7) gives

$$
\overline{[x]}^{a}=x_{0} \cdot a \cdot g_{x}^{-1} \cdot G_{x_{0}} \cdot a
$$


which is the first formula in (2.8). To show the second formula in (2.8) we iterate this argument, that is, we replace $g_{x}$ on the r.h.s. of $(2.9)$ with $a \cdot g_{x}^{-1} \cdot G_{x_{0}} \cdot a$. This gives

$$
\overline{\overline{[x]}}^{a}=x_{0} \cdot a \cdot\left(a \cdot g_{x}^{-1} \cdot G_{x_{0}} \cdot a\right)^{-1} \cdot G_{x_{0}} \cdot a=x_{0} \cdot g_{x} \cdot a^{-1} \cdot G_{x_{0}} \cdot a,
$$

which concludes the proof.

Remark 2.5. It is clear from the proof of Lemma 2.4 that any odd (resp. even) number of conjugations, defined by iterating (2.7), will reproduce the first (resp. the second) set in (2.8). Thus there is no need to go beyond the second conjugate class.

In the following proposition we find $\left(x_{0}, a\right)$ from Definition 2.3 for which distinct points from $\left[x_{0}\right]_{G}$ give rise to distinct conjugate and second conjugate classes.

Proposition 2.6. Let $\left(x_{0}, a\right)$ be as in Definition 2.3 and suppose that $a^{-1} \cdot G_{x_{0}} \cdot a \subset$ $G_{x_{0}}$. Then, for all $g \in G$, the following equivalence relations hold:

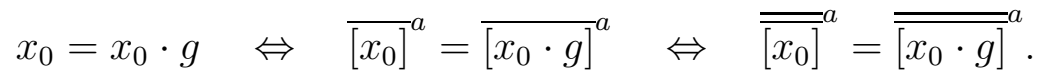

Proof. We use Lemma 2.4 to prove the relations in (2.11). It is clear that $x_{0}=x_{0} \cdot g$ implies the other two equalities. As for the opposite implications, let us first suppose that ${\overline{\left[x_{0}\right]}}^{a}={\overline{\left[x_{0} \cdot g\right]}}^{a}$, i.e., $\left[x_{0} \cdot a^{2}\right]_{\left(a^{-1} \cdot G_{x_{0}} \cdot a\right)}=\left[x_{0} \cdot a \cdot g^{-1} \cdot a\right]_{\left(a^{-1} \cdot G_{x_{0}} \cdot a\right)}$, for some $g \in G$. In other words,

$$
x_{0} \cdot a^{2}=x_{0} \cdot a \cdot g^{-1} \cdot g_{0} \cdot a \quad \text { for some } \quad g_{0} \in G_{x_{0}} .
$$

Hence, $a \cdot g^{-1} \cdot g_{0} \cdot a^{-1}=g_{0}^{\prime}$ for some $g_{0}^{\prime} \in G_{x_{0}}$ and therefore $g=g_{0} \cdot a^{-1} \cdot\left(g_{0}^{\prime}\right)^{-1} \cdot a$. Since $a^{-1} \cdot G_{x_{0}} \cdot a \subset G_{x_{0}}$, we obtain that $g \in G_{x_{0}}$.

Let us now suppose that ${\overline{\overline{\left[x_{0}\right]}}}^{a}={\overline{\overline{\left[x_{0} \cdot g\right]}}}^{a}$, that is, $\left[x_{0}\right]_{a^{-1} \cdot G_{x_{0}} \cdot a}=\left[x_{0} \cdot g\right]_{a^{-1} \cdot G_{x_{0}} \cdot a}$ for some $g \in G$. This means

$$
x_{0}=x_{0} \cdot g \cdot a^{-1} \cdot g_{0} \cdot a \quad \text { for some } \quad g_{0} \in G_{x_{0}} .
$$

Thus $g \cdot a^{-1} \cdot g_{0} \cdot a \in G_{x_{0}}$, i.e., $g=g_{0}^{\prime} \cdot a^{-1} \cdot g_{0}^{-1} \cdot a$ for some $g_{0}^{\prime} \in G_{x_{0}}$. Since $a^{-1} \cdot G_{x_{0}} \cdot a \subset G_{x_{0}}$, we obtain again that $g \in G_{x_{0}}$.

In the next theorem, which can be considered our main abstract result, we identify $\left(x_{0}, a\right)$ from Definition 2.3 for which distinct points from $\left[x_{0}\right]_{G}$ may give rise to coinciding conjugate and second conjugate classes.

Theorem 2.7. Let $S \subset G$ be a subgroup and set $R_{0}:=G_{x_{0}} \cap S$. Then, for all $a \in N_{G}\left(R_{0}, S\right)$ and $s \in S$,

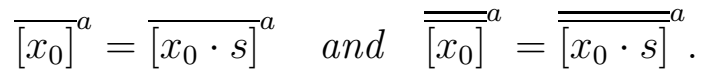

Furthermore, $\left[x_{0} \cdot g\right]_{S} \subset{\overline{\overline{\left[x_{0} \cdot g\right]}}}^{a}$ for all $g \in G$.

Remark 2.8. Clearly, the statement of Theorem 2.7 remains true if $R_{0}$ is replaced by any subgroup $R \subset R_{0}$ and the proof below pertains to this case. Moreover, as we will see in Subsection 4.3, the assumptions of Theorem 2.7 are compatible with $x_{0} \neq x_{0} \cdot s$ for some $s \in S$. That is, under these assumptions the first equivalence relation in (2.11) is not true. 
Proof. Concerning the first equality, we write using Lemma 2.4

$$
{\overline{\left[x_{0} \cdot s\right]}}^{a}=\left[x_{0} \cdot a \cdot s^{-1} \cdot a\right]_{a^{-1} \cdot G_{x_{0}} \cdot a}=\left[x_{0} \cdot r \cdot a^{2}\right]_{a^{-1} \cdot G_{x_{0}} \cdot a}={\overline{\left[x_{0}\right]}}^{a},
$$

where in the second step we noted that $r:=a \cdot s^{-1} a^{-1} \in R$ since $a \in N_{G}(R, S)$ and in the third step we used that $R \subset G_{x_{0}}$.

As for the second equality, we proceed similarly. Lemma 2.4 gives

$$
{\overline{\overline{\left[x_{0} \cdot s\right]}}}^{a}=\left[x_{0} \cdot s\right]_{a^{-1} \cdot G_{x_{0}} \cdot a}=\left[x_{0} \cdot a^{-1} \cdot r^{-1} \cdot a\right]_{a^{-1} \cdot G_{x_{0}} \cdot a}={\overline{\overline{\left[x_{0}\right]}}}^{a} \text {, }
$$

where we made use of the fact that $r^{-1}:=a \cdot s \cdot a^{-1} \in R \subset G_{x_{0}}$ and thus $r^{-1} \cdot G_{x_{0}}=G_{x_{0}}$.

To prove the last statement, we write for any $s^{\prime} \in S$

$$
x_{0} \cdot g \cdot s^{\prime}=x_{0} \cdot g \cdot a^{-1} \cdot\left(a \cdot s^{\prime} \cdot a^{-1}\right) \cdot a=x_{0} \cdot g \cdot a^{-1} \cdot r^{\prime} \cdot a \subset\left[x_{0} \cdot g\right]_{a^{-1} \cdot G_{x_{0}} \cdot a},
$$

where we used that $r^{\prime}=a \cdot s^{\prime} \cdot a^{-1} \in R \subset G_{x_{0}}$. By applying Lemma 2.4 we conclude the proof.

\subsection{Application in representation theory of $C^{*}$-algebras}

Let $\mathfrak{A}$ be a $C^{*}$-algebra and let $R \subset S \subset G$ be subgroups of the group $\operatorname{Aut}(\mathfrak{A})$ of automorphisms of $\mathfrak{A}$. Furthermore, we denote by $\operatorname{In}(\mathfrak{A}) \subset \operatorname{Aut}(\mathfrak{A})$ the normal subgroup of inner automorphisms. We denote by $P_{\mathfrak{A}} \subset \mathfrak{A}^{*}$ the set of pure states on $\mathfrak{A}$ on which $\operatorname{Aut}(\mathfrak{A})$ acts in a natural manner. For the resulting action of $G \subset \operatorname{Aut}(\mathfrak{A})$ we write

$$
P_{\mathfrak{A}} \times G \ni(\omega, \gamma) \rightarrow \omega \circ \gamma \in P_{\mathfrak{A}}
$$

In the spirit of [BR14, Definition 4.1], we define the set of sectors as $X_{\mathfrak{A}}:=$ $P_{\mathfrak{A}} / \operatorname{In}(\mathfrak{A})$. We recall in Proposition A.2, that for any $\omega_{1}, \omega_{2} \in P_{\mathfrak{A}}$, the equality of sectors $\left[\omega_{1}\right]_{\operatorname{In}(\mathfrak{A})}=\left[\omega_{2}\right]_{\operatorname{In}(\mathfrak{A})}$ holds iff the GNS representations of $\omega_{1}, \omega_{2}$ are unitarily equivalent. Furthermore, for any $\omega \in P_{\mathfrak{A}}$ the stabilizer group $G_{[\omega]_{\operatorname{In}(\mathfrak{A})}}$ is precisely the group of automorphisms from $G$ which are unitarily implementable in the GNS representation of $\omega$.

Since $\operatorname{In}(\mathfrak{A}) \subset \operatorname{Aut}(\mathfrak{A})$ is a normal subgroup, (2.18) gives rise to an action of $G$ on the space of sectors:

$$
X_{\mathfrak{A}} \times G \ni\left([\omega]_{\operatorname{In}(\mathfrak{A})}, \gamma\right) \rightarrow[\omega \circ \gamma]_{\operatorname{In}(\mathfrak{A})} \in X_{\mathfrak{A}}
$$

Let us now fix a reference ('vacuum') state $\omega_{\text {vac }} \in P_{\mathfrak{A}}$ and a reference ('background') automorphism $\alpha \in G$. The pair $\left(\left[\omega_{\text {vac }}\right]_{\operatorname{In}(\mathfrak{A})}, \alpha\right) \in P_{\mathfrak{A}} \times G$ will play the role of $\left(x_{0}, a\right)$ from Definition 2.3. Now Proposition 2.6 and Theorem 2.7 give the following:

Theorem 2.9. Let $\left(\left[\omega_{\mathrm{vac}}\right] \operatorname{In}(\mathfrak{A}), \alpha\right)$ and $R \subset S \subset G \subset \operatorname{Aut}(\mathfrak{A})$ be as above and $R \subset G_{\left[\omega_{\operatorname{vac}}\right]_{\operatorname{In}(\mathfrak{A})}}$.

(a) Suppose that $\alpha^{-1} \circ G_{\left[\omega_{\mathrm{vac}}\right]_{\operatorname{In}(\mathfrak{A})}} \circ \alpha \subset G_{\left[\omega_{\mathrm{vac}}\right]_{\operatorname{In}(\mathfrak{L})}}$. Then, for all $\gamma \in G$, the condition $\left[\omega_{\mathrm{vac}}\right]_{\operatorname{In}(\mathfrak{A})}=\left[\omega_{\mathrm{vac}} \circ \gamma\right]_{\operatorname{In}(\mathfrak{A})}$ is equivalent to any of the following two equalities:

$$
{\overline{\left[\left[\omega_{\mathrm{vac}}\right] \operatorname{In}(\mathfrak{A})\right.}}^{\alpha}={\overline{\left[\left[\omega_{\mathrm{vac}} \circ \gamma\right]_{\operatorname{In}(\mathfrak{A})}\right]}}^{\alpha}, \quad{\overline{\overline{\left[\left[\omega_{\mathrm{vac}}\right] \operatorname{In}(\mathfrak{A})\right.}}}^{\alpha}={\overline{\overline{\left[\left[\omega_{\mathrm{vac}} \circ \gamma\right]_{\operatorname{In}(\mathfrak{A})}\right.}}}^{\alpha} .
$$


(b) Suppose that $\alpha \in N_{G}(R, S)$. Then for all $\gamma \in S$

$$
{\overline{\left[\left[\omega_{\text {vac }}\right]_{\operatorname{In}(\mathfrak{l})}\right]}}^{\alpha}={\overline{\left[\left[\omega_{\text {vac }} \circ \gamma\right]_{\operatorname{In}(\mathfrak{l})}\right.}}^{\alpha} \text { and }{\overline{\overline{\left[\left[\omega_{\text {vac }}\right]_{\operatorname{In}(\mathfrak{l})}\right]}}}^{\alpha}={\overline{\overline{\left[\omega_{\text {vac }} \circ \gamma\right]_{\operatorname{In}(\mathfrak{l})}}}}^{\alpha} \text { holds. }
$$

(c) Suppose that $\alpha \in N_{G}(R, S)$. Then, for all $\gamma \in G$, $\left[\omega_{\mathrm{vac}} \circ \gamma\right]_{\operatorname{In}(\mathfrak{A}) \cdot S} \subset{\overline{\left[\left[\omega_{\mathrm{vac}} \circ \gamma\right]_{\operatorname{In}(\mathfrak{l l})}\right.}}^{\alpha}$.

As indicated in Remark 2.8, part (b) of this theorem is only non-trivial if $S \backslash G_{\left[\omega_{\mathrm{vac}}\right]_{\mathrm{In}(2)}} \neq$ $\emptyset$. This latter relation fails, in particular, under the combined assumptions of (a) and

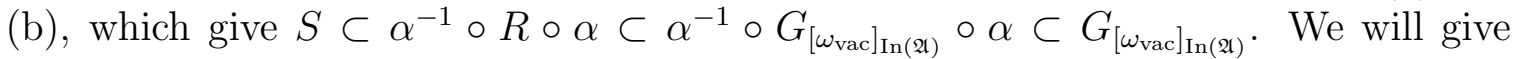
examples illustrating the non-trivial case in Subsection 4.3.

\section{Relative normalizers in the theory of canonical commutation relations}

\subsection{Relative normalizers in the inhomogeneous symplectic group}

Given an infinite dimensional real vector space $\mathcal{L}$ we denote its algebraic dual by $\mathcal{L}^{*}$ and the action of an element $\boldsymbol{v} \in \mathcal{L}^{*}$ on $\boldsymbol{f} \in \mathcal{L}$ by $(\boldsymbol{v}, \boldsymbol{f})$. For any real linear map $T: \mathcal{L} \rightarrow \mathcal{L}$ its transposition $T^{t}: \mathcal{L}^{*} \rightarrow \mathcal{L}^{*}$ is defined by $\left(T^{t} \boldsymbol{v}, \boldsymbol{f}\right)=(\boldsymbol{v}, T \boldsymbol{f})$. The group of invertible liner maps on $\mathcal{L}$ is denoted $\operatorname{GL}(\mathcal{L})$. We equip the vector space $\mathcal{L}$ with a non-degenerate symplectic form $\boldsymbol{\sigma}(\cdot, \cdot)$ and say that $T \in \mathrm{GL}(\mathcal{L})$ is symplectic if $\boldsymbol{\sigma}\left(T \boldsymbol{f}_{1}, T \boldsymbol{f}_{2}\right)=\boldsymbol{\sigma}\left(\boldsymbol{f}_{1}, \boldsymbol{f}_{2}\right)$ for all $\boldsymbol{f}_{1}, \boldsymbol{f}_{2} \in \mathcal{L}$. The group of symplectic maps on $\mathcal{L}$ is denoted $\operatorname{Sp}(\mathcal{L})$. We define the inhomogeneous symplectic group in a way which is suitable for our purposes, namely

$$
\operatorname{ISp}(\mathcal{L}):=\mathcal{L}^{*} \rtimes_{\varphi} \operatorname{Sp}(\mathcal{L})
$$

with the group homomorphism $\varphi: \operatorname{Sp}(\mathcal{L}) \rightarrow \operatorname{GL}\left(\mathcal{L}^{*}\right)$ given by $\varphi(T)=\left(T^{-1}\right)^{t}$. The elements of $\operatorname{ISp}(\mathcal{L})$ are pairs $g=(\boldsymbol{v}, T) \in \mathcal{L}^{*} \times \operatorname{GL}(\mathcal{L})$ and the product is defined by $\left(\boldsymbol{v}_{1}, T_{1}\right) \cdot\left(\boldsymbol{v}_{2}, T_{2}\right)=\left(\boldsymbol{v}_{1}+\left(T_{1}^{-1}\right)^{t} \boldsymbol{v}_{2}, T_{1} T_{2}\right)$. We write $T:=(0, T), \boldsymbol{v}:=(\boldsymbol{v}, I)$ and treat $\mathcal{L}^{*}$ and $\operatorname{Sp}(\mathcal{L})$ as subgroups of $\operatorname{ISp}(\mathcal{L})$. In this spirit we write

$$
\mathcal{L}_{\mathrm{R}}^{*} \subset \mathcal{L}_{\mathrm{S}}^{*} \subset \operatorname{ISp}(\mathcal{L}),
$$

for subspaces $\mathcal{L}_{\mathrm{R}}^{*}, \mathcal{L}_{\mathrm{S}}^{*} \subset \mathcal{L}^{*}$ which we treat as abelian subgroups of $\operatorname{ISp}(\mathcal{L})$. Since the group relations give $T \cdot \boldsymbol{v} \cdot T^{-1}=\left(T^{-1}\right)^{t} \boldsymbol{v}$, for all $T \in \operatorname{Sp}(\mathcal{L}), \boldsymbol{v} \in \mathcal{L}^{*}$, we immediately obtain:

Lemma 3.1. $T \in N_{\mathrm{ISp}(\mathcal{L})}\left(\mathcal{L}_{\mathrm{R}}^{*}, \mathcal{L}_{\mathrm{S}}^{*}\right)$ iff $\left(T^{-1}\right)^{t} \mathcal{L}_{\mathrm{S}}^{*} \subset \mathcal{L}_{\mathrm{R}}^{*}$.

For the above considerations $\operatorname{Sp}(\mathcal{L})$ could be replaced with any other subgroup of $\mathrm{GL}(\mathcal{L})$, but the symplectic structure will be important in the next subsection. 


\subsection{Relative normalizers in automorphism groups of CCR algebras}

Let us first summarize some relevant information from the theory of CCR algebras and their Bogoliubov automorphisms referring to [DG, Ru78, Ro70] for more complete treatment. The $C^{*}$-algebra $\mathfrak{A}$ of canonical commutation relations, associated with $\mathcal{L}$, is constructed in a standard manner: The $*$-algebra generated by symbols $\{W(\boldsymbol{f})\}_{\boldsymbol{f} \in \mathcal{L}}$ satisfying the Weyl relations

$$
W\left(\boldsymbol{f}_{1}\right) W\left(\boldsymbol{f}_{2}\right)=e^{-i \boldsymbol{\sigma}\left(\boldsymbol{f}_{1}, \boldsymbol{f}_{2}\right)} W\left(\boldsymbol{f}_{1}+\boldsymbol{f}_{2}\right), \quad W(\boldsymbol{f})^{*}=W(-\boldsymbol{f})
$$

is completed in the $C^{*}$-norm $\|\cdot\|=\sup _{(\pi, \mathcal{H})}\|\pi(\cdot)\|_{B(\mathcal{H})}$, where the supremum is taken over all representations $(\pi, \mathcal{H})$.

Proceeding to relevant automorphisms of $\mathfrak{A}$, we consider a group homomorphism $\alpha: \operatorname{ISp}(\mathcal{L}) \rightarrow \operatorname{Aut}(\mathfrak{A})$ defined on the Weyl operators by

$$
\alpha_{(\boldsymbol{v}, T)}(W(\boldsymbol{f}))=e^{-2 i(\boldsymbol{v}, T \boldsymbol{f})} W(T \boldsymbol{f}) .
$$

It is extended to $\mathfrak{A}$ using the uniqueness theorem of Slawny, see [Pe89, Theorem 2.1 and page 13], and the boundedness of automorphisms of $C^{*}$-algebras [BR, Proposition 2.3.1].

Lemmas 2.2 and 3.1 give the following criterion for the existence of non-empty relative normalizers for subgroups of $\operatorname{Aut}(\mathfrak{A})$.

Lemma 3.2. Consider the abelian subgroups $\mathcal{L}_{\mathrm{R}}^{*} \subset \mathcal{L}_{\mathrm{S}}^{*} \subset \operatorname{ISp}(\mathcal{L})$ as in (3.2). Let $R:=\alpha_{\mathcal{L}_{\mathrm{R}}^{*}}, S:=\alpha_{\mathcal{L}_{\mathrm{S}}^{*}}$ and $G \subset \operatorname{Aut}(\mathfrak{A})$ be any subgroup containing $\alpha_{\mathrm{ISp}(\mathcal{L})}$. Then for any $T \in \operatorname{ISp}(\mathcal{L})$ the following implication holds:

$$
\left(T^{-1}\right)^{t} \mathcal{L}_{\mathrm{S}}^{*} \subset \mathcal{L}_{\mathrm{R}}^{*} \quad \Rightarrow \quad \alpha_{T} \in N_{G}(R, S) .
$$

To be able to apply Theorem 2.9 we need to choose the 'vacuum' state $\omega_{\text {vac }}$. We do it in the standard manner: Suppose that $\mathcal{L}$ is a dense subspace of a Hilbert space $\mathfrak{h}$ with a scalar product $\langle\cdot, \cdot\rangle$ such that $\boldsymbol{\sigma}(\cdot, \cdot)=\operatorname{Im}\langle\cdot, \cdot\rangle$ and define on the Weyl operators

$$
\omega_{\text {vac }}(W(\boldsymbol{f})):=e^{-\frac{1}{2}\|\boldsymbol{f}\|^{2}}, \quad \boldsymbol{f} \in \mathcal{L} .
$$

The resulting GNS representation $\pi_{\text {vac }}$, which can be chosen to act on the Fock space $\mathcal{F}=\Gamma(\mathfrak{h})$ (see (4.4) below), is faithful and irreducible. We have

$$
W_{\mathrm{vac}}(\boldsymbol{f}):=\pi_{\mathrm{vac}}(W(\boldsymbol{f}))=e^{a^{*}(\boldsymbol{f})-a(\boldsymbol{f})},
$$

where $a^{*}(\cdot), a(\cdot)$ are the creation and annihilation operators on $\mathcal{F}$. A concrete choice of $\mathfrak{h}$ will be made in the next section. 


\section{Infravacua in QED as elements of relative nor- malizers}

\subsection{The model}

We consider one spinless non-relativistic electron interacting with the second-quantized electromagnetic field in the setting of non-relativistic quantum electrodynamics (QED). For a textbook presentation see [Sp], we follow here mostly [CFP09].

We set $L^{2}\left(\mathbb{R}^{3} ; \mathbb{C}^{3}\right):=L^{2}\left(\mathbb{R}^{3}\right) \otimes \mathbb{C}^{3}$ and denote the scalar product by $\langle\cdot, \cdot\rangle$. The single-photon Hilbert space $\mathfrak{h}$ is the following space of transverse functions

$$
L_{\mathrm{tr}}^{2}\left(\mathbb{R}^{3} ; \mathbb{C}^{3}\right):=\left\{\boldsymbol{f} \in L^{2}\left(\mathbb{R}^{3} ; \mathbb{C}^{3}\right) \mid \boldsymbol{k} \cdot \boldsymbol{f}(\boldsymbol{k})=0 \text { a.e. }\right\}
$$

and we denote by $P_{\mathrm{tr}}: L^{2}\left(\mathbb{R}^{3} ; \mathbb{C}^{3}\right) \rightarrow L^{2}\left(\mathbb{R}^{3} ; \mathbb{C}^{3}\right)$ the orthogonal projection on $L_{\mathrm{tr}}^{2}\left(\mathbb{R}^{3} ; \mathbb{C}^{3}\right)$. We write $S^{2}$ for the unit sphere in $\mathbb{R}^{3}$ and introduce the polarisation vectors $\mathbb{R}^{3} \ni$ $\boldsymbol{k} \mapsto \boldsymbol{\epsilon}_{ \pm}(\boldsymbol{k}) \in S^{2}$, given by, e.g., [LL04]

$$
\boldsymbol{\epsilon}_{+}(\boldsymbol{k})=\frac{\left(k_{2},-k_{1}, 0\right)}{\sqrt{k_{1}^{2}+k_{2}^{2}}}, \quad \boldsymbol{\epsilon}_{-}(\boldsymbol{k})=\frac{\boldsymbol{k}}{|\boldsymbol{k}|} \times \boldsymbol{\epsilon}_{+}(\boldsymbol{k}),
$$

which satisfy $\boldsymbol{k} \cdot \boldsymbol{\epsilon}_{ \pm}(\boldsymbol{k})=0$ and $\boldsymbol{\epsilon}_{+}(\boldsymbol{k}) \cdot \boldsymbol{\epsilon}_{-}(\boldsymbol{k})=0$ for $\boldsymbol{k}=\left(k_{1}, k_{2}, k_{3}\right) \in \mathbb{R}^{3}$. In terms of these vectors we can write, in the $L^{2}$-sense,

$$
\left(P_{\mathrm{tr}} \boldsymbol{f}\right)(\boldsymbol{k})=\sum_{\lambda= \pm}\left(\boldsymbol{f}(\boldsymbol{k}) \cdot \boldsymbol{\epsilon}_{\lambda}(\boldsymbol{k})\right) \boldsymbol{\epsilon}_{\lambda}(\boldsymbol{k})
$$

Next, we denote by $\mathcal{F}$ the symmetric Fock space over $\mathfrak{h}=L_{\mathrm{tr}}^{2}\left(\mathbb{R}^{3} ; \mathbb{C}^{3}\right)$, which is the fiber Fock space pertaining to the decomposition (1.5). More precisely,

$$
\mathcal{F}:=\oplus_{n=0}^{\infty} \mathcal{F}^{(n)}, \quad \mathcal{F}^{(n)}:=\operatorname{Sym}_{n}\left(\mathfrak{h}^{\otimes n}\right), \quad \mathcal{F}^{(0)}=\mathbb{C} \Omega .
$$

We define the quantized electromagnetic vector potential ${ }^{3}$ with infrared and ultraviolet cut-offs $0 \leq \sigma \leq \kappa$ as an operator on a certain domain in $\mathcal{F}$

$$
\boldsymbol{A}_{[\sigma, \kappa]}(\boldsymbol{x}):=\sum_{\lambda= \pm} \int \frac{d^{3} k}{\sqrt{|\boldsymbol{k}|}} \chi_{[\sigma, \kappa]}(|\boldsymbol{k}|) \boldsymbol{\epsilon}_{\lambda}(\boldsymbol{k})\left(e^{-i \boldsymbol{k} \cdot \boldsymbol{x}} a_{\lambda}^{*}(\boldsymbol{k})+e^{i \boldsymbol{k} \cdot \boldsymbol{x}} a_{\lambda}(\boldsymbol{k})\right) .
$$

Here $\chi_{\Delta}$ denotes the characteristic function of a set $\Delta$ and $a_{\lambda}(\boldsymbol{k}), a_{\lambda}^{*}(\boldsymbol{k})$ are the standard (improper) creation and annihilation operators on $\mathcal{F}$ such that $\left[a_{\lambda}(\boldsymbol{k}), a_{\lambda^{\prime}}^{*}\left(\boldsymbol{k}^{\prime}\right)\right]=$ $\delta_{\lambda \lambda^{\prime}} \delta\left(\boldsymbol{k}-\boldsymbol{k}^{\prime}\right)$ and all other commutators vanish. They are related to the creation and annihilation operators appearing in $(3.7)$ via $a^{*}(\boldsymbol{f})=\sum_{\lambda= \pm} \int d^{3} k a_{\lambda}^{*}(\boldsymbol{k})\left(\boldsymbol{\epsilon}_{\lambda}(\boldsymbol{k}) \cdot \boldsymbol{f}(\boldsymbol{k})\right)$, for $f \in \mathfrak{h}$.

Furthermore, we define the free Hamiltonian and momentum operators of the electromagnetic field

$$
H_{\mathrm{ph}}=\sum_{\lambda= \pm} \int d^{3} k|\boldsymbol{k}| a_{\lambda}^{*}(\boldsymbol{k}) a_{\lambda}(\boldsymbol{k}), \quad \boldsymbol{P}_{\mathrm{ph}}=\sum_{\lambda= \pm} \int d^{3} k \boldsymbol{k} a_{\lambda}^{*}(\boldsymbol{k}) a_{\lambda}(\boldsymbol{k}) .
$$

\footnotetext{
${ }^{3}$ up to a normalization constant, which is absorbed into $\tilde{\alpha}^{1 / 2}$ in (4.7).
} 
The fiber Hamiltonians, which appeared in the decomposition (1.5), are given by

$$
H_{\boldsymbol{P}, \sigma}=\frac{1}{2}\left(\boldsymbol{P}-\boldsymbol{P}_{\mathrm{ph}}+\tilde{\alpha}^{1 / 2} \boldsymbol{A}_{[\sigma, \kappa]}(0)\right)^{2}+H_{\mathrm{ph}}, \quad H_{\boldsymbol{P}}:=H_{\boldsymbol{P}, \sigma=0} .
$$

They are self-adjoint, positive operators on a common domain independent of $\boldsymbol{P}$ (see, e.g., [Sp, Hi00, KM14]). We denote by $E_{\boldsymbol{P}, \sigma}:=\inf \operatorname{Spec}\left(H_{\boldsymbol{P}, \sigma}\right), E_{\boldsymbol{P}}:=\inf \operatorname{Spec}\left(H_{\boldsymbol{P}}\right)$ the respective infima of the spectra of $H_{\boldsymbol{P}, \sigma}, H_{\boldsymbol{P}}$. They are rotation invariant functions of $\boldsymbol{P}$.

Now we recall some spectral results, mostly from [CFP09, FP10], which we will use below. From now on we discuss the regime of low coupling $\tilde{\alpha}$ and we are interested in momenta $\boldsymbol{P}$ restricted to the ball

$$
\mathcal{S}=\left\{\boldsymbol{P} \in \mathbb{R}^{3}|| \boldsymbol{P} \mid<\frac{1}{3}\right\}
$$

It is well known that for any $\sigma>0$ the operators $H_{\boldsymbol{P}, \sigma}$ have ground-states $\Psi_{\boldsymbol{P}, \sigma} \in \mathcal{F}$, $\left\|\Psi_{\boldsymbol{P}, \sigma}\right\|=1$, corresponding to isolated eigenvalues $E_{\boldsymbol{P}, \sigma}$. The dependence $\boldsymbol{P} \mapsto E_{\boldsymbol{P}, \sigma}$ is analytic for any fixed $\sigma>0$ by Kato perturbation theory. In the limit $\sigma \rightarrow 0$, as the spectral gap closes, $\Psi_{\boldsymbol{P}, \sigma}$ tend weakly to zero [CFP09, Fr73, Fr74, Ch08] and the Hamiltonians $H_{\boldsymbol{P}}$ do not have ground-states for $\boldsymbol{P} \neq 0$ [HH08]. To analyze this phenomenon, one introduces auxiliary vectors

$$
\Phi_{\boldsymbol{P}, \sigma}:=W_{\mathrm{vac}}\left(\boldsymbol{v}_{\boldsymbol{P}, \sigma}\right) \Psi_{\boldsymbol{P}, \sigma}, \quad W_{\mathrm{vac}}\left(\boldsymbol{v}_{\boldsymbol{P}, \sigma}\right)=e^{a^{*}\left(\boldsymbol{v}_{\boldsymbol{P}, \sigma}\right)-a\left(\boldsymbol{v}_{\boldsymbol{P}, \sigma}\right)},
$$

where $\boldsymbol{v}_{\boldsymbol{P}, \sigma}$ has the form

$$
\boldsymbol{v}_{\boldsymbol{P}, \sigma}(\boldsymbol{k})=\tilde{\alpha}^{1 / 2} P_{\operatorname{tr}} \frac{\chi_{[\sigma, \kappa]}(|\boldsymbol{k}|)}{|\boldsymbol{k}|^{3 / 2}} \frac{\nabla E_{\boldsymbol{P}, \sigma}}{1-\hat{k} \cdot \nabla E_{\boldsymbol{P}, \sigma}},
$$

and we set $\hat{\boldsymbol{k}}:=\boldsymbol{k} /|\boldsymbol{k}|$ and $\nabla E_{\boldsymbol{P}, \sigma}:=\nabla_{\boldsymbol{P}} E_{\boldsymbol{P}, \sigma}$. The following lemma collects some facts from $[\mathrm{CFP} 09, \mathrm{FP} 10]{ }^{4}$

Lemma 4.1. Let $\tilde{\alpha}>0$ be sufficiently small and $\boldsymbol{P} \in \mathcal{S}$. Then

(a) The function $\boldsymbol{P} \mapsto E_{\boldsymbol{P}}$ is rotation invariant, twice differentiable and has strictly positive second derivative with respect to $|\boldsymbol{P}|$.

(b) $\lim _{\sigma \rightarrow 0} \partial_{\boldsymbol{P}}^{\beta} E_{\boldsymbol{P}, \sigma}$ exists and equals $\partial_{\boldsymbol{P}}^{\beta} E_{\boldsymbol{P}}$ for $|\beta| \leq 2$.

(c) $\left|\nabla E_{\boldsymbol{P}, \sigma}\right| \leq v_{\max }<1$ and $\left|\nabla E_{\boldsymbol{P}}\right| \leq v_{\max }<1$ for some constant $v_{\max }$, uniformly in $\sigma$ and in $\boldsymbol{P} \in \mathcal{S}$.

(d) $\Phi_{\boldsymbol{P}}:=\lim _{\sigma \rightarrow 0} \Phi_{\boldsymbol{P}, \sigma}$ exists in norm for a suitable choice of the phases of $\Psi_{\boldsymbol{P}, \sigma}$.

In the following we assume that the phases of $\Psi_{\boldsymbol{P}, \sigma}$ are fixed as in Lemma 4.1 (d). Using Lemma 4.1 (b) we can define the pointwise limit

$$
\boldsymbol{v}_{\boldsymbol{P}}(\boldsymbol{k}):=\lim _{\sigma \rightarrow 0} \boldsymbol{v}_{\boldsymbol{P}, \sigma}(\boldsymbol{k})=\frac{\tilde{\alpha}^{1 / 2}}{|\boldsymbol{k}|^{3 / 2}} P_{\operatorname{tr}} \chi_{[0, \kappa]}(|k|) \frac{\nabla E_{\boldsymbol{P}}}{1-\hat{\boldsymbol{k}} \cdot \nabla E_{\boldsymbol{P}}} .
$$

\footnotetext{
${ }^{4}$ Precisely, for (a) and (b) see [FP10, Theorem III.3 and Corollary III.4], for (c) see [CFP09, Eq. (III.2) and formula (V.6)] and for (d) [CFP09, Theorem III.1].
} 
We note that the expressions $1-\hat{k} \cdot \nabla E_{\boldsymbol{P}, \sigma}$ and $1-\hat{k} \cdot \nabla E_{\boldsymbol{P}}$ in the denominators of (4.10) and (4.11) are different from zero by Lemma 4.1 (c). The fact that $\boldsymbol{v}_{\boldsymbol{P}}$ is not in $L_{\mathrm{tr}}^{2}\left(\mathbb{R}^{3} ; \mathbb{C}^{3}\right)$ for $0 \neq \boldsymbol{P} \in \mathcal{S}$ will be important below. We will also use that $\boldsymbol{v}_{\boldsymbol{P}=0}=0$, which is a consequence of rotational invariance.

\subsection{Infravacua as elements of relative normalizers}

In this subsection we will give a concrete realization of the structure $\mathcal{L}_{\mathrm{R}}^{*} \subset \mathcal{L}_{\mathrm{S}}^{*} \subset \operatorname{ISp}(\mathcal{L})$ which appeared in (3.2). The symplectic space $\mathcal{L}$, which we will use in the following analysis, is defined as follows:

$$
\mathcal{L}:=\bigcup_{\varepsilon>0} L_{\mathrm{tr}, \varepsilon}^{2}\left(\mathbb{R}^{3} ; \mathbb{C}^{3}\right)
$$

where $L_{\mathrm{tr}, \varepsilon}^{2}\left(\mathbb{R}^{3} ; \mathbb{C}^{3}\right):=\left\{\boldsymbol{f} \in L_{\mathrm{tr}}^{2}\left(\mathbb{R}^{3} ; \mathbb{C}^{3}\right) \mid \boldsymbol{f}(\boldsymbol{k})=0\right.$ for $\left.|\boldsymbol{k}| \leq \varepsilon\right\}$. The symplectic form is given by $\boldsymbol{\sigma}(\cdot, \cdot)=\operatorname{Im}\langle\cdot, \cdot\rangle$. We introduce the following subspaces of $\mathcal{L}^{*}$,

$$
\mathcal{L}_{\mathrm{S}}^{*}:=\mathcal{L}_{\mathrm{D}}^{*}+\mathcal{L}_{\mathrm{R}}^{*}, \text { where } \mathcal{L}_{\mathrm{D}}^{*}:=\operatorname{Span}_{\mathbb{R}}\left\{\boldsymbol{v}_{\boldsymbol{P}} \mid \boldsymbol{P} \in \mathcal{S}\right\}, \quad \mathcal{L}_{\mathrm{R}}^{*}:=L_{\mathrm{tr}}^{2}\left(\mathbb{R}^{3} ; \mathbb{C}^{3}\right)_{\mathbb{R}},
$$

and the dressing functions $\boldsymbol{v}_{\boldsymbol{P}}$ appeared in (4.11). Here $L_{\mathrm{tr}}^{2}\left(\mathbb{R}^{3} ; \mathbb{C}^{3}\right)_{\mathbb{R}}$ denotes the subspace of real-valued functions in $L_{\mathrm{tr}}^{2}\left(\mathbb{R}^{3} ; \mathbb{C}^{3}\right)$ and the linear spans in (4.13) are over the field of real numbers. Furthermore, we set here $(\boldsymbol{v}, \boldsymbol{f}):=\operatorname{Im}\langle\boldsymbol{v}, \boldsymbol{f}\rangle$ for $\boldsymbol{v} \in \mathcal{L}_{\mathrm{S}}^{*}$, $\boldsymbol{f} \in \mathcal{L}$, which is well-defined since all $\boldsymbol{f} \in \mathcal{L}$ vanish near zero.

In the following we exhibit maps $T \in \operatorname{Sp}(\mathcal{L})$ such that $\left(T^{-1}\right)^{t} \mathcal{L}_{\mathrm{S}}^{*} \subset \mathcal{L}_{\mathrm{R}}^{*}$. By Lemmas 3.1, 3.2 such maps, and the corresponding automorphisms $\alpha_{T}$, are elements of relative normalizers. More precisely, we will show in Theorem 4.3, that infravacuum maps first introduced by Kraus, Polley and Reents [Re74, KPR77, Kr82] have the above mapping property. For this purpose, in Proposition 4.2 we collect the essential features of the infravacuum maps which can be found in the literature, up to some technical mismatches relating, e.g., to different choices of the symplectic space and different implementation of the infrared regularization. We postpone the somewhat lengthy definition of the Kraus-Polley-Reents maps and the proof of Proposition 4.2 to Section 5. At the same time our proof of part (a) of this proposition is new and easier to follow than the corresponding arguments available in [Re74, KPR77, Kr82, Ku98].

Proposition 4.2. There exist $T \in \operatorname{Sp}(\mathcal{L})$ such that:

(a) The limit $T \boldsymbol{v}_{\boldsymbol{P}}:=\lim _{n \rightarrow \infty} T \boldsymbol{v}_{\boldsymbol{P}, \sigma_{n}}$ exists in $L_{\mathrm{tr}}^{2}\left(\mathbb{R}^{3} ; \mathbb{C}^{3}\right)$ for a certain subsequence $\left\{\sigma_{n}\right\}_{n \in \mathbb{N}}$ tending to zero.

(b) $\|T \boldsymbol{f}\| \leq c\|\boldsymbol{f}\|$ for all real-valued $\boldsymbol{f} \in \mathcal{L}$ and some $c$ independent of $\boldsymbol{f}$.

Such $T$ are called infravacuum maps.

As noted below formula (4.11), the functions $\boldsymbol{v}_{\boldsymbol{P}, \sigma}$ escape from $L_{\mathrm{tr}}^{2}\left(\mathbb{R}^{3} ; \mathbb{C}^{3}\right)$ in the limit $\sigma \rightarrow 0$. Therefore $T \boldsymbol{v}_{\boldsymbol{P}}$ above should be understood as one symbol and part (a) of Proposition 4.2 does not follow from part (b). Instead, it demonstrates a remarkable regularizing property of $T$. We stress that this feature is restricted to real-valued 
functions and an infravacuum map cannot be complex-linear. This is a consequence of the symplectic property and the following computation

$$
\operatorname{Im}\left\langle T \boldsymbol{v}_{\boldsymbol{P}, \sigma_{n}}, T\left(i \boldsymbol{v}_{\boldsymbol{P}, \sigma_{n}}\right)\right\rangle=\operatorname{Im}\left\langle\boldsymbol{v}_{\boldsymbol{P}, \sigma_{n}}, i \boldsymbol{v}_{\boldsymbol{P}, \sigma_{n}}\right\rangle=\left\|\boldsymbol{v}_{\boldsymbol{P}, \sigma_{n}}\right\|^{2} \underset{n \rightarrow \infty}{\rightarrow} \infty
$$

which shows that $T\left(i \boldsymbol{v}_{\boldsymbol{P}, \sigma_{n}}\right)$ diverges in $L_{\mathrm{tr}}^{2}\left(\mathbb{R}^{3} ; \mathbb{C}^{3}\right)$ as $n \rightarrow \infty$. Another consequence of properties (a), (b) from Proposition 4.2 is that the infravacuum maps are elements of relative normalizers, which is the main result of this section.

Theorem 4.3. The infravacuum maps $T \in \operatorname{Sp}(\mathcal{L})$ from Proposition 4.2 satisfy

$$
\left(T^{-1}\right)^{t} \mathcal{L}_{\mathrm{S}}^{*} \subset \mathcal{L}_{\mathrm{R}}^{*}
$$

where the subspaces $\mathcal{L}_{\mathrm{R}}^{*} \subset \mathcal{L}_{\mathrm{S}}^{*}$ are given by (4.13). Hence $T \in N_{\mathrm{ISp}(\mathcal{L})}\left(\mathcal{L}_{\mathrm{R}}^{*}, \mathcal{L}_{\mathrm{S}}^{*}\right)$ and $\alpha_{T} \in N_{G}(R, S)$. Here $R:=\alpha_{\mathcal{L}_{\mathrm{R}}^{*}}, S:=\alpha_{\mathcal{L}_{\mathrm{S}}^{*}}$ and $G \subset \operatorname{Aut}(\mathfrak{A})$ is any subgroup containing $\alpha_{\operatorname{ISp}(\mathcal{L})}$. The homomorphism $\alpha$ is given by (3.4).

Proof. A general element $\boldsymbol{v}_{\mathrm{S}} \in \mathcal{L}_{\mathrm{S}}^{*}$ has the form $\boldsymbol{v}_{\mathrm{S}}=\boldsymbol{v}_{\mathrm{D}}+\boldsymbol{v}_{\mathrm{R}}$, where $\boldsymbol{v}_{\mathrm{D}} \in \mathcal{L}_{\mathrm{D}}^{*}$ and $\boldsymbol{v}_{\mathrm{R}} \in \mathcal{L}_{\mathrm{R}}^{*}:=L_{\mathrm{tr}}^{2}\left(\mathbb{R}^{3} ; \mathbb{C}^{3}\right)_{\mathbb{R}}$. Thus we have for any $\boldsymbol{f} \in \mathcal{L}$

$$
\left(\left(T^{-1}\right)^{t} \boldsymbol{v}_{\mathrm{S}}, \boldsymbol{f}\right)=\left(\boldsymbol{v}_{\mathrm{S}}, T^{-1} \boldsymbol{f}\right)=\operatorname{Im}\left\langle\boldsymbol{v}_{\mathrm{D}}, T^{-1} \boldsymbol{f}\right\rangle+\operatorname{Im}\left\langle\boldsymbol{v}_{\mathrm{R}}, T^{-1} \boldsymbol{f}\right\rangle .
$$

Clearly, we have $\boldsymbol{v}_{\mathrm{D}}=\sum_{i=1}^{N} c_{i} \boldsymbol{v}_{\boldsymbol{P}_{i}}$ for some $c_{i} \in \mathbb{R}, N \in \mathbb{N}, \boldsymbol{P}_{i} \in \mathcal{S}$. We define accordingly its approximant $\boldsymbol{v}_{\mathrm{D}, \sigma}:=\sum_{i=1}^{N} c_{i} \boldsymbol{v}_{\boldsymbol{P}_{i}, \sigma} \in \mathcal{L}$ and write, using the sequence $\left\{\sigma_{n}\right\}_{n \in \mathbb{N}}$ from Proposition 4.2,

$$
\operatorname{Im}\left\langle\boldsymbol{v}_{\mathrm{D}}, T^{-1} \boldsymbol{f}\right\rangle=\lim _{n \rightarrow \infty} \operatorname{Im}\left\langle\boldsymbol{v}_{\mathrm{D}, \sigma_{n}}, T^{-1} \boldsymbol{f}\right\rangle=\lim _{n \rightarrow \infty} \operatorname{Im}\left\langle T \boldsymbol{v}_{\mathrm{D}, \sigma_{n}}, \boldsymbol{f}\right\rangle=\left(T \boldsymbol{v}_{\mathrm{D}}, \boldsymbol{f}\right) .
$$

Here in the first step we used Lemma 4.1 (b) and the fact that $T^{-1} \boldsymbol{f}$ vanishes in some neighbourhood of zero. In the second step we used that $T$ is symplectic and in the last step we applied Proposition 4.2 (a). This latter statement also tells us that $T \boldsymbol{v}_{\mathrm{D}}:=\lim _{n \rightarrow \infty} T \boldsymbol{v}_{\mathrm{D}, \sigma_{n}} \in \mathcal{L}_{\mathrm{R}}^{*}$.

The second term on the right hand side of (4.16) is handled by a similar and simpler consideration: We define $\boldsymbol{v}_{\mathrm{R}}^{\sigma}(\boldsymbol{k}):=\chi_{[\sigma, \infty)}(|\boldsymbol{k}|) \boldsymbol{v}_{\mathrm{R}}(\boldsymbol{k})$ so that $\boldsymbol{v}_{\mathrm{R}}=\lim _{\sigma \rightarrow 0} \boldsymbol{v}_{\mathrm{R}}^{\sigma}$ in the norm topology of $L_{\mathrm{tr}}^{2}\left(\mathbb{R}^{3} ; \mathbb{C}^{3}\right)$. Since the functions $\boldsymbol{v}_{\mathrm{R}}^{\sigma}$ are real-valued, Proposition 4.2 (b) gives the existence of $T \boldsymbol{v}_{\mathrm{R}}:=\lim _{\sigma \rightarrow 0} T \boldsymbol{v}_{\mathrm{R}}^{\sigma} \in \mathcal{L}_{\mathrm{R}}^{*}$. Now the proof of (4.15) is completed by a computation analogous to (4.17).

The last statement of the theorem is a consequence of Lemmas 3.1, 3.2.

\subsection{Infravacua and velocity superselection}

Proceeding towards the problem of velocity superselection, we define the following states on the Weyl algebra $\mathfrak{A}$ over the symplectic space $\mathcal{L}$ as introduced in (4.12).

$$
\omega_{\boldsymbol{P}}(A):=\lim _{\sigma \rightarrow 0}\left\langle\Psi_{\boldsymbol{P}, \sigma}, \pi_{\mathrm{vac}}(A) \Psi_{\boldsymbol{P}, \sigma}\right\rangle=\left\langle\Phi_{\boldsymbol{P}}, \pi_{\mathrm{vac}}\left(\alpha_{\boldsymbol{v}_{\boldsymbol{P}}}(A)\right) \Phi_{\boldsymbol{P}}\right\rangle, \quad A \in \mathfrak{A} .
$$

These states describe plane-wave configurations of the electron with velocity $\nabla E_{\boldsymbol{P}}$. Here $\pi_{\mathrm{vac}}$ is the vacuum representation defined in (3.6) and in the second step we used 
(4.9), the Weyl relations (3.3), Lemma 4.1 (d), definition (3.4) and the specifications $(\boldsymbol{v}, \boldsymbol{f})=\operatorname{Im}\langle\boldsymbol{v}, \boldsymbol{f}\rangle, \boldsymbol{\sigma}\left(\boldsymbol{f}_{1}, \boldsymbol{f}_{2}\right)=\operatorname{Im}\left\langle\boldsymbol{f}_{1}, \boldsymbol{f}_{2}\right\rangle$ for $\boldsymbol{v} \in \mathcal{L}_{\mathrm{S}}^{*}, \boldsymbol{f}, \boldsymbol{f}_{1}, \boldsymbol{f}_{2} \in \mathcal{L}$, which appeared below (4.13) and above (3.6), respectively. As $\omega_{\boldsymbol{P}}$ are pure states on $\mathfrak{A}$ (cf. [KR, Corollary 10.2.5]), we can use the framework of Subsection 2.3 to study the corresponding superselection structure. The starting point is the following proposition, whose proof is given in Section 6 .

Proposition 4.4. We have $\left[\omega_{\boldsymbol{P}}\right]_{\operatorname{In}(\mathfrak{l})} \neq\left[\omega_{\boldsymbol{P}^{\prime}}\right]_{\operatorname{In}(\mathfrak{A})}$ for all $\boldsymbol{P}, \boldsymbol{P}^{\prime} \in \mathcal{S}$ such that $\boldsymbol{P} \neq \boldsymbol{P}^{\prime}$.

We recall that for the present model, and a similar model describing the electron with spin, disjointness of $\left[\omega_{\boldsymbol{P}=0}\right]_{\operatorname{In}(\mathfrak{A})}$ and $\left[\omega_{\boldsymbol{P}^{\prime}}\right]_{\operatorname{In}(\mathfrak{A l})}, \boldsymbol{P}^{\prime} \neq 0$ was shown in [CFP09,CF07] by exploiting the absence of the number operator in non-Fock representations. In the Nelson model and in a semi-relativistic model of QED disjointness for all $\boldsymbol{P} \neq \boldsymbol{P}^{\prime}$ (from suitable balls around zero) was verified in [Fr73, KM14] using theory of infinite tensor products of Hilbert spaces. Our proof in Section 6, inspired by [Ku98, Lemma $2.2]$, exhibits central sequences in $\mathfrak{A}$ which can distinguish $\left[\omega_{\boldsymbol{P}}\right]_{\operatorname{In}(\mathfrak{A})}$ from $\left[\omega_{\boldsymbol{P}^{\prime}}\right]_{\operatorname{In}(\mathfrak{l})}$. We think this argument is quite simple and intuitive.

Our main result, concerning the problem of velocity superselection in the model of non-relativistic QED, is stated below. The conjugate classes are defined using an arbitrary subgroup $\alpha_{\operatorname{ISp}(\mathcal{L})} \subset G \subset \operatorname{Aut}(\mathfrak{A})$, the Fock vacuum $\left[\omega_{\operatorname{vac}}\right]_{\operatorname{In}(\mathfrak{l})}$ as the reference sector and two different types of background automorphisms: In part (a) we consider 'regular' backgrounds in which case the conjugation procedure does not merge the disjoint sectors from Proposition 4.4 into coinciding classes. In part (b) we show that such a merging effect is achieved, if the infravacuum automorphisms are used as a background.

Theorem 4.5. For the family of states $\left\{\omega_{\boldsymbol{P}}\right\}_{\boldsymbol{P} \in \mathcal{S}}$ defined in (4.18) the following is true:

(a) Suppose that $\beta \in G \subset \operatorname{Aut}(\mathfrak{A})$ is unitarily implemented in $\pi_{\text {vac }}$ (e.g., $\beta=\mathrm{id}$ ). Then, for all $0 \neq \boldsymbol{P}^{\prime} \in \mathcal{S}$,

$$
{\overline{\left[\left[\omega_{\boldsymbol{P}=0}\right] \operatorname{In}(\mathfrak{l})\right.}}^{\beta} \neq{\overline{\left[\left[\omega_{\boldsymbol{P}^{\prime}}\right]_{\operatorname{In}(\mathfrak{l})}\right.}}^{\beta} \text { and }{\overline{\left[\left[\omega_{\boldsymbol{P}=0}\right]_{\operatorname{In}(\mathfrak{l})}\right.}}^{\beta} \neq{\overline{\overline{\left.\left[\omega_{\boldsymbol{P}^{\prime}}\right]_{\operatorname{In}(\mathfrak{l})}\right]}}}^{\beta} \text { holds. }
$$

(b) Let $T \in \operatorname{Sp}(\mathcal{L})$ be an infravacuum map (cf. Proposition 4.2) and $\alpha_{T} \in \operatorname{Aut}(\mathfrak{A})$ be given by (3.4). Then, for all $\boldsymbol{P}, \boldsymbol{P}^{\prime} \in \mathcal{S}$,

$$
{\overline{\left[\left[\omega_{\boldsymbol{P}}\right]_{\operatorname{In}(\mathfrak{l})}\right]}}^{\alpha_{T}}={\overline{\left[\left[\omega_{\boldsymbol{P}^{\prime}}\right]_{\operatorname{In}(\mathfrak{l} l)}\right.}}^{\alpha_{T}} \text { and }{\overline{\overline{\left[\left[\omega_{\boldsymbol{P}}\right]_{\operatorname{In}(\mathfrak{l})}\right]}}}^{\alpha_{T}}={\overline{\overline{\left[\omega_{\boldsymbol{P}^{\prime}}\right]_{\operatorname{In}(\mathfrak{l})}}}}^{\alpha_{T}} \text { holds. }
$$

Proof. We consider the subspaces $\mathcal{L}_{\mathrm{R}}^{*}, \mathcal{L}_{\mathrm{S}}^{*}$ defined in (4.13), set $R:=\alpha_{\mathcal{L}_{\mathrm{R}}^{*}}, S:=\alpha_{\mathcal{L}_{\mathrm{S}}^{*}}$ and fix $\alpha_{\mathrm{ISp}(\mathcal{L})} \subset G \subset \operatorname{Aut}(\mathfrak{A})$ as in Theorem 4.3. Now parts (a) and (b) can be inferred from the corresponding parts of Theorem 2.9 as follows:

(a) We recall that $G_{\left[\omega_{\mathrm{vac}}\right]_{\operatorname{In}(\mathfrak{H})}}$ coincides with the group of automorphisms from $G$ which are unitarily implemented in the vacuum representation (cf. Theorem A.2),

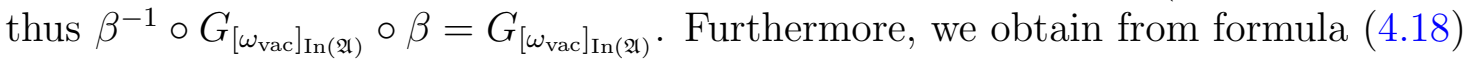

$$
\omega_{\boldsymbol{P}}(A)=\left\langle\Omega, \pi_{\mathrm{vac}}\left(U_{\boldsymbol{P}} \alpha_{\boldsymbol{v}_{\boldsymbol{P}}}(A) U_{\boldsymbol{P}}^{*}\right) \Omega\right\rangle=\left\langle\Omega, \pi_{\mathrm{vac}}\left(\alpha_{\boldsymbol{v}_{\boldsymbol{P}}}\left(\tilde{U}_{\boldsymbol{P}} A \tilde{U}_{\boldsymbol{P}}^{*}\right)\right) \Omega\right\rangle, \quad A \in \mathfrak{A} .
$$


Here we found a unitary $U_{\boldsymbol{P}} \in \mathfrak{A}$ such that $\Phi_{\boldsymbol{P}}=\pi_{\text {vac }}\left(U_{\boldsymbol{P}}^{*}\right) \Omega(\mathrm{cf}$. [KR, Theorem 10.2.1]) and set $\tilde{U}_{\boldsymbol{P}}:=\alpha_{\boldsymbol{v}_{\boldsymbol{P}}}^{-1}\left(U_{\boldsymbol{P}}\right)$. Therefore,

$$
\left[\omega_{\boldsymbol{P}}\right]_{\operatorname{In}(\mathfrak{A})}=\left[\omega_{\text {vac }} \circ \alpha_{\boldsymbol{v}_{\boldsymbol{P}}}\right]_{\operatorname{In}(\mathfrak{A})} \quad \text { and } \quad\left[\omega_{\boldsymbol{P}=0}\right]_{\operatorname{In}(\mathfrak{A})}=\left[\omega_{\text {vac }}\right]_{\operatorname{In}(\mathfrak{A})},
$$

where the second equality follows from $\boldsymbol{v}_{\boldsymbol{P}=0}=0$ (cf. definition (4.11)). Given these identifications, the statement follows from Proposition 4.4 and Theorem 2.9 (a).

(b) By Theorem 4.3, we have $\alpha_{T} \in N_{G}(R, S)$. Now, since $\alpha_{\boldsymbol{v}_{\boldsymbol{P}}} \in S$, the first equality in (4.22) and Theorem 2.9 (b) give the claim. The statement concerning the second conjugate classes can also be obtained from Theorem 2.9 (c) noting that $\left[\omega_{\boldsymbol{P}}\right]_{\operatorname{In}(\mathfrak{A}) \cdot S}=\left[\omega_{\text {vac }}\right]_{\operatorname{In}(\mathfrak{A}) \cdot S}$ for any $\boldsymbol{P} \in \mathcal{S}$.

Theorem 4.5 (b) shows that the effect of velocity superselection is eliminated at the level of the conjugate classes with respect to the infravacuum background. It turns out that the infravacuum automorphisms can also be used to cure velocity superselection at the level of states: By formula (4.18) we can write $\omega_{\boldsymbol{P}}=\omega_{\Phi_{\boldsymbol{P}}} \circ \alpha_{\boldsymbol{v}_{\boldsymbol{P}}}$, where we set $\omega_{\Phi}(\cdot):=\left\langle\Phi, \pi_{\text {vac }}(\cdot) \Phi\right\rangle$ for any unit vector $\Phi \in \mathcal{F}$. We define

$$
\omega_{\boldsymbol{P}, T}:=\omega_{\Phi_{\boldsymbol{P}}} \circ \alpha_{T} \circ \alpha_{\boldsymbol{v}_{\boldsymbol{P}}}
$$

where $T$ is an infravacuum map and $\boldsymbol{P} \in \mathcal{S}$. These are modifications of the states $\omega_{\boldsymbol{P}}$ above by inserting the infravacuum between the state $\omega_{\Phi_{P}}$ of the 'undressed electron' and the automorphism $\alpha_{\boldsymbol{v}_{\boldsymbol{P}}}$ constructed from the dressing transformation. As we show below, all $\omega_{\boldsymbol{P}, T}$ lie in the same sector and hence the corresponding GNS representations are unitarily equivalent. Thus there is no velocity superselection in this situation.

Theorem 4.6. Let $T$ be an infravacuum map. For all $\boldsymbol{P}, \boldsymbol{P}^{\prime} \in \mathcal{S}$, we have $\left[\omega_{\boldsymbol{P}, T}\right]_{\operatorname{In}(\mathfrak{A})}=$ $\left[\omega_{\boldsymbol{P}^{\prime}, T}\right] \operatorname{In}(\mathfrak{A})$.

Proof. Since for any unit vector $\Phi \in \mathcal{F}$ we can find $i_{\Phi} \in \operatorname{In}(\mathfrak{A})$ such that $\omega_{\Phi}=\omega_{\text {vac }} \circ i_{\Phi}$, [KR, Theorem 10.2.1], we write

$$
\left[\omega_{\boldsymbol{P}, T}\right]_{\operatorname{In}(\mathfrak{A})}=\left[\omega_{\mathrm{vac}}\right]_{\operatorname{In}(\mathfrak{A})} \circ \alpha_{T} \circ \alpha_{\boldsymbol{v}_{\boldsymbol{P}}}=\left[\omega_{\mathrm{vac}}\right]_{\operatorname{In}(\mathfrak{A})} \circ \alpha_{\left(T^{-1}\right)^{t} \boldsymbol{v}_{\boldsymbol{P}}} \circ \alpha_{T}=\left[\omega_{\mathrm{vac}}\right]_{\operatorname{In}(\mathfrak{A})} \circ \alpha_{T},
$$

where in the second step we used the homomorphism property of $\alpha$ (cf. (3.4)) and the multiplication law in $\operatorname{ISp}(\mathcal{L})$ which gives $T \cdot \boldsymbol{v}_{\boldsymbol{P}} \cdot T^{-1}=\left(T^{-1}\right)^{t} \boldsymbol{v}_{\boldsymbol{P}}$. In the third step we used Theorem 4.3 which ensures that $\left(T^{-1}\right)^{t} \boldsymbol{v}_{\boldsymbol{P}} \in L_{\mathrm{tr}}^{2}\left(\mathbb{R}^{3} ; \mathbb{C}^{3}\right)$ and thus $\alpha_{\left(T^{-1}\right)^{t} \boldsymbol{v}_{\boldsymbol{P}}} \in$ $G_{\left[\omega_{\text {vac }}\right]_{\operatorname{In}(\mathfrak{A})}}$ by the last statement of Proposition A.2.

As a consequence of Theorem 4.6, we have ${\overline{\left[\left[\omega_{\boldsymbol{P}, T}\right] \operatorname{In}(\mathfrak{A})\right.}}^{\alpha_{T}}={\overline{\left[\omega_{\boldsymbol{P}^{\prime}, T}\right] \operatorname{In}(\mathfrak{A})}}^{\alpha_{T}}$ for all $\boldsymbol{P} \neq \boldsymbol{P}^{\prime}$. In fact, since $\left[\omega_{\boldsymbol{P}, T}\right]_{\operatorname{In}(\mathfrak{A})}$ is independent of $\boldsymbol{P}$ by the above result, it is clear from Definition 2.3 that $G_{\omega_{\boldsymbol{P}, T}, \omega_{\mathrm{vac}}}^{\alpha_{T}}$ is independent of $\boldsymbol{P}$ as well, which implies the same for $\left.\left[\overline{[\omega}_{\boldsymbol{P}, T}\right]_{\operatorname{In}(\mathfrak{A})}\right]^{\alpha_{T}}$.

As the states (4.23) rely on the non-canonical decomposition of the single-electron state $\omega_{\boldsymbol{P}}$ into the 'undressed electron' $\omega_{\Phi_{\boldsymbol{P}}}$ and the 'dressing' $\alpha_{\boldsymbol{v}_{\boldsymbol{P}}}$, their physical realization appears difficult, even as a thought experiment. To improve on this, one could consider the following family of states, which does not rely on such a decomposition:

$$
\omega_{\boldsymbol{P}}^{T}:=\omega_{\boldsymbol{P}} \circ \alpha_{T}=\omega_{\Phi_{\boldsymbol{P}}} \circ \alpha_{\boldsymbol{v}_{\boldsymbol{P}}} \circ \alpha_{T} .
$$


However, velocity superselection persists for these states, as shown below: The sectors $\left[\omega_{\boldsymbol{P}}^{T}\right]_{\operatorname{In}(\mathfrak{A})}$ depend on $\boldsymbol{P}$, and correspondingly, the GNS representations are not equivalent. It is clear from the proof below that replacing $\alpha_{T}$ in (4.25) with any other automorphism of $\mathfrak{A}$ will not improve the situation. Thus the modification (4.23) is the only possibility we can see to cure velocity superselection at the level of states with the help of the infravacuum.

Proposition 4.7. Let $T$ be an infravacuum map. Then, $\left[\omega_{\boldsymbol{P}}^{T}\right]_{\operatorname{In}(\mathfrak{A})} \neq\left[\omega_{\boldsymbol{P}^{\prime}}^{T}\right]_{\operatorname{In}(\mathfrak{A})}$ for all $\boldsymbol{P}, \boldsymbol{P}^{\prime} \in \mathcal{S}$ such that $\boldsymbol{P} \neq \boldsymbol{P}^{\prime}$.

Proof. We argue by contradiction: Suppose $\left[\omega_{\boldsymbol{P}}^{T}\right]_{\operatorname{In}(\mathfrak{A})}=\left[\omega_{\boldsymbol{P}^{\prime}}^{T}\right]_{\operatorname{In}(\mathfrak{l})}$ for some $\boldsymbol{P} \neq \boldsymbol{P}^{\prime}$ from $\mathcal{S}$. This means $\left[\omega_{\boldsymbol{P}}\right]_{\operatorname{In}(\mathfrak{A})} \circ \alpha_{T}=\left[\omega_{\boldsymbol{P}^{\prime}}\right]_{\operatorname{In}(\mathfrak{A})} \circ \alpha_{T}$ in conflict with Proposition 4.4.

\section{Kraus-Polley-Reents infravacua}

In this section we provide a proof of Proposition 4.2.

First, we introduce the decomposition of functions from $\mathcal{L}=\bigcup_{\varepsilon>0} L_{\mathrm{tr}, \varepsilon}^{2}\left(\mathbb{R}^{3} ; \mathbb{C}^{3}\right)$ into radial and angular parts

$$
L_{\mathrm{tr}, \varepsilon}^{2}\left(\mathbb{R}^{3} ; \mathbb{C}^{3}\right)=L_{\varepsilon}^{2}\left(\mathbb{R}_{+}\right) \otimes L_{\mathrm{tr}}^{2}\left(S^{2} ; \mathbb{C}^{3}\right)
$$

Here $L_{\varepsilon}^{2}\left(\mathbb{R}_{+}\right)$is the space of radial functions with measure $|\boldsymbol{k}|^{2} d|\boldsymbol{k}|$ vanishing for $|\boldsymbol{k}| \leq \varepsilon$ and we write $L^{2}\left(\mathbb{R}_{+}\right):=L_{\varepsilon=0}^{2}\left(\mathbb{R}_{+}\right)$. Further, $L_{\mathrm{tr}}^{2}\left(S^{2} ; \mathbb{C}^{3}\right)$ is the space of transverse angular functions with the natural spherical measure $d \Omega(\theta, \phi):=\sin \theta d \theta d \phi$. The latter space is the range of the projection $P^{\operatorname{tr}}: L^{2}\left(S^{2} ; \mathbb{C}^{3}\right) \rightarrow L^{2}\left(S^{2} ; \mathbb{C}^{3}\right)$ given by

$$
\left(P^{\operatorname{tr}} \boldsymbol{f}\right)(\hat{\boldsymbol{k}}):=\sum_{\lambda= \pm}\left(\boldsymbol{f}(\hat{\boldsymbol{k}}) \cdot \boldsymbol{\epsilon}_{\lambda}(\hat{\boldsymbol{k}})\right) \boldsymbol{\epsilon}_{\lambda}(\hat{\boldsymbol{k}})
$$

As in [KPR77], we introduce the transverse, vector valued spherical harmonics for $\ell \neq 0,-\ell \leq m \leq \ell$

$$
\boldsymbol{Y}_{\ell m \pm}=\frac{1}{\sqrt{\ell(\ell+1)}} \boldsymbol{a}_{ \pm} Y_{\ell m} \quad \text { with } \quad \boldsymbol{a}_{+}=|\boldsymbol{k}| \nabla_{\boldsymbol{k}} \quad \text { and } \quad \boldsymbol{a}_{-}=\hat{\boldsymbol{k}} \times \boldsymbol{a}_{+}
$$

which are elements of $L_{\mathrm{tr}}^{2}\left(S^{2} ; \mathbb{C}^{3}\right)$. Here $Y_{\ell m}$ are the usual spherical harmonics which form an orthonormal basis in $L^{2}\left(S^{2}\right)$ with measure $d \Omega$. As a consequence, $Y_{\ell m \pm}$ with $\ell \neq 0$ form a complete basis of $L_{\mathrm{tr}}^{2}\left(S^{2} ; \mathbb{C}^{3}\right)$. Furthermore, $J^{2} \boldsymbol{Y}_{\ell m \pm}=\ell(\ell+1) \boldsymbol{Y}_{\ell m \pm}$, where $\boldsymbol{J}=\boldsymbol{L}+\boldsymbol{S}$ is the total angular momentum of photons including the orbital and spin part (the latter being a constant matrix on $\mathbb{C}^{3}$ ). These properties of $\boldsymbol{Y}_{\ell m \pm}$ are discussed more extensively in Appendix B.

Keeping the decomposition (5.1) in mind, we define the relevant symplectic map $T$.

Definition 5.1. The Kraus-Polley-Reents infravacuum map $T: \mathcal{L} \rightarrow \mathcal{L}$ is defined as follows ${ }^{5}$ :

\footnotetext{
${ }^{5}$ By small modifications of this definition one can easily obtain many different maps satisfying properties (a), (b) from Proposition 4.2. We restrict attention to the simplest choice.
} 
- We introduce sequences $\varepsilon_{i}:=2^{-(i-1)} \kappa$ and $b_{i}:=\frac{1}{i}$ for $i=1,2,3 \ldots$.

- We define functions $\xi_{i}(|\boldsymbol{k}|):=\frac{\chi_{\left[\varepsilon_{i+1}, \varepsilon_{i}\right]}(|\boldsymbol{k}|)}{|\boldsymbol{k}|^{3 / 2}} \in L^{2}\left(\mathbb{R}_{+}\right)$and their normalized counterparts $\tilde{\xi}_{i}(|\boldsymbol{k}|):=\xi_{i}(|\boldsymbol{k}|) /\left\|\xi_{i}\right\|_{L^{2}\left(\mathbb{R}_{+}\right)}$.

- We define the orthogonal projections $\boldsymbol{Q}_{i}: L_{\mathrm{tr}}^{2}\left(\mathbb{R}^{3} ; \mathbb{C}^{3}\right) \rightarrow L_{\mathrm{tr}}^{2}\left(\mathbb{R}^{3} ; \mathbb{C}^{3}\right)$ and $\tilde{\boldsymbol{Q}}_{i}$ : $L_{\mathrm{tr}}^{2}\left(S^{2} ; \mathbb{C}^{3}\right) \rightarrow L_{\mathrm{tr}}^{2}\left(S^{2} ; \mathbb{C}^{3}\right)$ given by

$$
\boldsymbol{Q}_{i}=\left|\tilde{\xi}_{i}\right\rangle\left\langle\tilde{\xi}_{i}\right| \otimes \tilde{\boldsymbol{Q}}_{i} \quad \text { with } \quad \tilde{\boldsymbol{Q}}_{i}:=\sum_{0<\ell \leq i} \sum_{m=-\ell}^{\ell} \sum_{\lambda= \pm}\left|\boldsymbol{Y}_{\ell m \lambda}\right\rangle\left\langle\boldsymbol{Y}_{\ell m \lambda}\right| .
$$

Since $\left|\tilde{\xi}_{i}\right\rangle\left\langle\tilde{\xi}_{i}\right|: L_{\varepsilon}^{2}\left(\mathbb{R}_{+}\right) \rightarrow L_{\varepsilon_{i+1}}^{2}\left(\mathbb{R}_{+}\right)$we have $\boldsymbol{Q}_{i}: \mathcal{L} \rightarrow \mathcal{L}$.

- We introduce the complex-linear maps $T_{1}, T_{2}: \mathcal{L} \rightarrow \mathcal{L}$

$$
T_{1}:=1+\underset{n \rightarrow \infty}{\mathrm{s}-\lim _{i=1}} \sum_{i=1}^{n}\left(b_{i}-1\right) \boldsymbol{Q}_{i}, \quad T_{2}:=1+\mathrm{s}_{n \rightarrow \infty} \lim _{i=1}^{n}\left(\frac{1}{b_{i}}-1\right) \boldsymbol{Q}_{i} .
$$

Clearly, the maps are well-defined, since $\boldsymbol{Q}_{i} \boldsymbol{f}=0$ for any $\boldsymbol{f} \in \mathcal{L}$ and sufficiently large $i$. We will denote by $T_{1, n}, T_{2, n}$ the respective approximants.

- Let $\hat{\Gamma}$ be the complex conjugation in momentum space. We introduce the reallinear map $T: \mathcal{L} \rightarrow \mathcal{L}$ given by

$$
T:=T_{1} \frac{1+\hat{\Gamma}}{2}+T_{2} \frac{1-\hat{\Gamma}}{2}
$$

and denote by $T_{n}$ the respective approximants. In other words, writing $\boldsymbol{f}=$ $\boldsymbol{f}_{1}+i \boldsymbol{f}_{2} \in \mathcal{L}$, where $\boldsymbol{f}_{1}, \boldsymbol{f}_{2}$ are real-valued, we have $T \boldsymbol{f}=T_{1} \boldsymbol{f}_{1}+i T_{2} \boldsymbol{f}_{2}$.

Following [KPR77, Ku98], we show that $T$ is symplectic and invertible. We also verify that $T_{1}$ extends to a bounded map on $L_{\mathrm{tr}}^{2}\left(\mathbb{R}^{3} ; \mathbb{C}^{3}\right)$ which yields Proposition 4.2 (b).

Lemma 5.2. We have for $\boldsymbol{f}_{0}, \boldsymbol{f}_{1}, \boldsymbol{f}_{2} \in \mathcal{L}$

$$
\left\langle T_{1} \boldsymbol{f}_{1}, T_{2} \boldsymbol{f}_{2}\right\rangle=\left\langle\boldsymbol{f}_{1}, \boldsymbol{f}_{2}\right\rangle, \quad T_{1} T_{2} \boldsymbol{f}_{0}=\boldsymbol{f}_{0}, \quad T_{2} T_{1} \boldsymbol{f}_{0}=\boldsymbol{f}_{0} .
$$

Furthermore, $T$ is symplectic and invertible with $T^{-1}=T_{2} \frac{1+\hat{\Gamma}}{2}+T_{1} \frac{1-\hat{\Gamma}}{2}$ and $T_{1}$ extends to a bounded operator on $L_{\mathrm{tr}}^{2}\left(\mathbb{R}^{3} ; \mathbb{C}^{3}\right)$.

Proof. Making use of self-adjointness of $T_{1, n}, T_{2, n}$ on $L_{\mathrm{tr}}^{2}\left(\mathbb{R}^{3} ; \mathbb{C}^{3}\right)$ and of the fact that $Q_{i}$ are mutually orthogonal projections, we have

$$
\left.\left\langle T_{1, n} \boldsymbol{f}_{1}, T_{2, n} \boldsymbol{f}_{2}\right\rangle=\left\langle\boldsymbol{f}_{1},\left(\mathbf{1}+\sum_{i^{\prime}=1}^{n}\left(b_{i^{\prime}}-1\right) \boldsymbol{Q}_{i^{\prime}}\right)\left(\mathbf{1}+\sum_{i=1}^{n}\left(\frac{1}{b_{i}}-1\right) \boldsymbol{Q}_{i}\right) \boldsymbol{f}_{2}\right)\right\rangle=\left\langle\boldsymbol{f}_{1}, \boldsymbol{f}_{2}\right\rangle
$$

This computation gives properties (5.7). Now choosing $\boldsymbol{f}, \boldsymbol{f}^{\prime} \in \mathcal{L}$ and decomposing them into real and imaginary parts as $\boldsymbol{f}=\boldsymbol{f}_{1}+i \boldsymbol{f}_{2}, \boldsymbol{f}^{\prime}=\boldsymbol{f}_{1}^{\prime}+i \boldsymbol{f}_{2}^{\prime}$, we have

$$
\operatorname{Im}\left\langle T_{1} \boldsymbol{f}_{1}+i T_{2} \boldsymbol{f}_{2}, T_{1} \boldsymbol{f}_{1}^{\prime}+i T_{2} \boldsymbol{f}_{2}^{\prime}\right\rangle=-\left\langle\boldsymbol{f}_{2}, \boldsymbol{f}_{1}^{\prime}\right\rangle+\left\langle\boldsymbol{f}_{1}, \boldsymbol{f}_{2}^{\prime}\right\rangle=\operatorname{Im}\left\langle\boldsymbol{f}, \boldsymbol{f}^{\prime}\right\rangle .
$$


Next, we set $\hat{T}:=T_{2} \frac{1+\hat{\Gamma}}{2}+T_{1} \frac{1-\hat{\Gamma}}{2}$ and check $T \hat{T} \boldsymbol{f}=\hat{T} T \boldsymbol{f}=\boldsymbol{f}$ using (5.7). Finally, the boundedness of $T_{1}$ follows from the computation

$$
\left\|\sum_{i=1}^{\infty}\left(b_{i}-1\right) \boldsymbol{Q}_{i} \boldsymbol{f}\right\|^{2} \leq C \sum_{i=1}^{n}\left\langle\boldsymbol{f}, \boldsymbol{Q}_{i} \boldsymbol{f}\right\rangle \leq C\|\boldsymbol{f}\|^{2},
$$

where we could choose a finite $n$ in the first step since $\boldsymbol{f} \in \mathcal{L}$. As $C$ is independent of $n$, this concludes the proof.

In preparation for the proof of Proposition 4.2 (a), we set $\sigma_{n}=\varepsilon_{n}, n \in \mathbb{N}$, where $\varepsilon_{n}$ appeared in Definition 5.1. Recalling definition (4.10) and setting as above $\xi_{i}(|\boldsymbol{k}|):=|\boldsymbol{k}|^{-3 / 2} \chi_{\left[\varepsilon_{i+1}, \varepsilon_{i}\right]}(|\boldsymbol{k}|)$, we write

$$
\boldsymbol{v}_{\boldsymbol{P}, \sigma_{n}}(\boldsymbol{k})=\sum_{i=1}^{n-1}\left(\xi_{i}(|\boldsymbol{k}|) \otimes \boldsymbol{\varphi}_{\boldsymbol{P}, n}^{\mathrm{tr}}(\hat{\boldsymbol{k}})\right)
$$

where

$$
\boldsymbol{\varphi}_{\boldsymbol{P}, n}^{\operatorname{tr}}(\hat{\boldsymbol{k}}):=P^{\operatorname{tr}} \boldsymbol{\varphi}_{\boldsymbol{P}, n}(\hat{\boldsymbol{k}}), \quad \boldsymbol{\varphi}_{\boldsymbol{P}, n}(\hat{\boldsymbol{k}}):=\tilde{\alpha}^{1 / 2} \frac{\nabla E_{\boldsymbol{P}, \sigma_{n}}}{1-\hat{\boldsymbol{k}} \cdot \nabla E_{\boldsymbol{P}, \sigma_{n}}} .
$$

We also define

$$
\boldsymbol{\varphi}_{\boldsymbol{P}}^{\operatorname{tr}}(\hat{\boldsymbol{k}}):=P^{\operatorname{tr}} \boldsymbol{\varphi}_{\boldsymbol{P}}(\hat{\boldsymbol{k}}), \quad \boldsymbol{\varphi}_{\boldsymbol{P}}(\hat{\boldsymbol{k}}):=\tilde{\alpha}^{1 / 2} \frac{\nabla E_{\boldsymbol{P}}}{1-\hat{\boldsymbol{k}} \cdot \nabla E_{\boldsymbol{P}}} .
$$

As a consequence of Lemma 4.1 and dominated convergence we have

$$
\lim _{n \rightarrow \infty}\left\|\varphi_{P, n}-\varphi_{P}\right\|_{L^{2}\left(S^{2} ; \mathbb{C}^{3}\right)}=0
$$

from which it also follows that $\lim _{n \rightarrow \infty}\left\|\varphi_{P, n}^{\mathrm{tr}}-\varphi_{P}^{\mathrm{tr}}\right\|_{L_{\mathrm{tr}}^{2}\left(S^{2} ; \mathbb{C}^{3}\right)}=0$ since $\left\|P^{\mathrm{tr}}\right\|=1$.

Since $\boldsymbol{v}_{\boldsymbol{P}, \sigma_{n}} \in \mathcal{L}$ are real-valued, we have $T \boldsymbol{v}_{\boldsymbol{P}, \sigma_{n}}=T_{1} \boldsymbol{v}_{\boldsymbol{P}, \sigma_{n}}$. By definition of $T_{1}$ and $\boldsymbol{Q}_{j}$, and by the support properties of $\xi_{i}$, we have

$$
T_{1} \boldsymbol{v}_{\boldsymbol{P}, \sigma_{n}}=\left(\mathbf{1}+\sum_{j=1}^{\infty}\left(b_{j}-1\right) \boldsymbol{Q}_{j}\right) \boldsymbol{v}_{\boldsymbol{P}, \sigma_{n}}=\sum_{i=1}^{n-1}\left(\mathbf{1}+\left(b_{i}-1\right) \boldsymbol{Q}_{i}\right)\left(\xi_{i} \otimes \boldsymbol{\varphi}_{\boldsymbol{P}, n}^{\mathrm{tr}}\right) .
$$

We set $\psi_{i, n}:=\left(1+\left(b_{i}-1\right) \boldsymbol{Q}_{i}\right)\left(\xi_{i} \otimes \boldsymbol{\varphi}_{\boldsymbol{P}, n}^{\mathrm{tr}}\right)$ and note that $\left\langle\psi_{i, n}, \psi_{i^{\prime}, n^{\prime}}\right\rangle_{L_{\mathrm{tr}}^{2}\left(\mathbb{R}^{3} ; \mathbb{C}^{3}\right)}=0$ for $i \neq i^{\prime}$ and any $n, n^{\prime} \in \mathbb{N}$. We also observe that by (5.14)

$$
\psi_{i}:=\lim _{n \rightarrow \infty} \psi_{i, n}=\left(1+\left(b_{i}-1\right) \boldsymbol{Q}_{i}\right)\left(\xi_{i} \otimes \boldsymbol{\varphi}_{\boldsymbol{P}}^{\operatorname{tr}}\right)
$$

exists in $L_{\mathrm{tr}}^{2}\left(\mathbb{R}^{3} ; \mathbb{C}^{3}\right)$. We want to show that the vector $T_{1} \boldsymbol{v}_{\boldsymbol{P}}:=\sum_{i=1}^{\infty} \psi_{i}$ exists in $L_{\mathrm{tr}}^{2}\left(\mathbb{R}^{3} ; \mathbb{C}^{3}\right)$, as it is a natural candidate for $\lim _{n \rightarrow \infty} T_{1} \boldsymbol{v}_{\boldsymbol{P}, \sigma_{n}}$. For this, we prove the following lemma.

Lemma 5.3. Fix $i \in \mathbb{N}$. Then there exists $C_{i}>0$ independent of $n$ such that

$$
\sup _{n \in \mathbb{N}}\left\|\psi_{i, n}\right\|_{L_{\mathrm{tr}}^{2}\left(\mathbb{R}^{3} ; \mathbb{C}^{3}\right)}^{2} \leq C_{i}, \quad \text { where } \sum_{i=1}^{\infty} C_{i}<\infty .
$$

In particular, $\sum_{i=1}^{\infty} \psi_{i, n}$ and $\sum_{i=1}^{\infty} \psi_{i}$ exist in $L_{\mathrm{tr}}^{2}\left(\mathbb{R}^{3} ; \mathbb{C}^{3}\right)$, and $\lim _{n \rightarrow \infty} \sum_{i=1}^{\infty} \psi_{i, n}=$ $\sum_{i=1}^{\infty} \psi_{i}$ in the same topology. 
Proof. To verify (5.17) we estimate the norm

$$
\begin{aligned}
\left\|\psi_{i, n}\right\|_{L_{\mathrm{tr}}^{2}\left(\mathbb{R}^{3} ; \mathbb{C}^{3}\right)}^{2} & =\left\|\xi_{i} \otimes\left(1+\left(b_{i}-1\right) \tilde{\boldsymbol{Q}}_{i}\right) \boldsymbol{\varphi}_{\boldsymbol{P}, n}^{\mathrm{tr}}\right\|_{L_{\mathrm{tr}}^{2}\left(\mathbb{R}^{3} ; \mathbb{C}^{3}\right)}^{2} \\
& =\left\|\xi_{i}\right\|_{L^{2}\left(\mathbb{R}_{+}\right)}^{2}\left\|\left(\mathbf{1}-\tilde{\boldsymbol{Q}}_{i}\right) \boldsymbol{\varphi}_{\boldsymbol{P}, n}^{\mathrm{tr}}+b_{i} \tilde{\boldsymbol{Q}}_{i} \boldsymbol{\varphi}_{\boldsymbol{P}, n}^{\operatorname{tr}}\right\|_{L_{\mathrm{tr}}^{2}\left(S^{2} ; \mathbb{C}^{3}\right)}^{2} \\
& \leq \ln \frac{\epsilon_{i}}{\epsilon_{i+1}}\left(\left\|\left(\mathbf{1}-\tilde{\boldsymbol{Q}}_{i}\right) \boldsymbol{\varphi}_{\boldsymbol{P}, n}^{\mathrm{tr}}\right\|_{L_{\mathrm{tr}}^{2}\left(S^{2} ; \mathbb{C}^{3}\right)}^{2}+b_{i}^{2}\left\|\boldsymbol{\varphi}_{\boldsymbol{P}, n}^{\mathrm{tr}}\right\|_{L_{\mathrm{tr}}^{2}\left(S^{2} ; \mathbb{C}^{3}\right)}^{2}\right) .
\end{aligned}
$$

For the last term, (5.14) gives $\left\|\varphi_{P, n}^{\mathrm{tr}}\right\|_{L_{\mathrm{tr}}^{2}\left(S^{2} ; \mathbb{C}^{3}\right)}^{2} \leq c_{1}$ uniformly in $n$.

Concerning the term with $\left(\mathbf{1}-\tilde{\boldsymbol{Q}}_{i}\right)$, we make use of the completeness of the vectorvalued spherical harmonics:

$$
\begin{aligned}
\left\|\left(1-\tilde{Q}_{i}\right) \varphi_{\boldsymbol{P}, n}^{\mathrm{tr}}\right\|_{L_{\mathrm{tr}}^{2}\left(S^{2} ; \mathbb{C}^{3}\right)}^{2} & =\sum_{\ell>i} \sum_{m=-\ell}^{\ell} \sum_{\lambda= \pm}\left|\left\langle Y_{\ell m \lambda}, \varphi_{\boldsymbol{P}, n}\right\rangle_{L^{2}\left(S^{2} ; \mathbb{C}^{3}\right)}\right|^{2} \\
& \leq \frac{2}{i^{2}}\left(\left\langle\varphi_{\boldsymbol{P}, n}, \boldsymbol{L}^{2} \boldsymbol{\varphi}_{\boldsymbol{P}, n}\right\rangle_{L^{2}\left(S^{2} ; \mathbb{C}^{3}\right)}+2\left\|\boldsymbol{\varphi}_{\boldsymbol{P}, n}\right\|_{L^{2}\left(S^{2} ; \mathbb{C}^{3}\right)}^{2}\right)
\end{aligned}
$$

where $\boldsymbol{\varphi}_{\boldsymbol{P}, n}$ is defined in (5.13) and we replaced $\boldsymbol{\varphi}_{\boldsymbol{P}, n}^{\mathrm{tr}}$ with $\boldsymbol{\varphi}_{\boldsymbol{P}, n}$, which is justified since $Y_{\ell m \lambda}$ are transverse. In the last step we exploited $J^{2} Y_{\ell m \pm}=\ell(\ell+1) Y_{\ell m \pm}$, inserted $\boldsymbol{J}^{2}=(\boldsymbol{L}+\boldsymbol{S})^{2}$, applied the Cauchy-Schwarz inequality to terms of the form $L_{i} S_{i}$ with $i=1,2,3$, and used $\left\|\boldsymbol{S}^{2}\right\|=2$. Here $\left\|\boldsymbol{\varphi}_{\boldsymbol{P}, n}\right\|_{L^{2}\left(S^{2} ; \mathbb{C}^{3}\right)}^{2}$ is uniformly bounded in $n$ due to (5.14). To estimate the first term in (5.19) we write $\boldsymbol{L}^{2}=-\frac{1}{\sin \theta} \frac{\partial}{\partial \theta}\left(\sin \theta \frac{\partial}{\partial \theta}\right)-\frac{1}{\sin ^{2} \theta} \frac{\partial^{2}}{\partial \phi^{2}}$ in spherical coordinates, choosing our reference frame in $\mathbb{R}^{3}$ such that $\nabla E_{\boldsymbol{P}, \sigma_{n}}$ is in the direction of the third axis, and compute

$$
\begin{aligned}
& \left\langle\boldsymbol{\varphi}_{\boldsymbol{P}, n}, \boldsymbol{L}^{2} \boldsymbol{\varphi}_{\boldsymbol{P}, n}\right\rangle_{L^{2}\left(S^{2} ; \mathbb{C}^{3}\right)} \\
& =-2 \pi \tilde{\alpha}\left|\nabla E_{\boldsymbol{P}, \sigma_{n}}\right|^{2} \int_{-1}^{1} \frac{d \cos \theta}{1-\cos \theta\left|\nabla E_{\boldsymbol{P}, \sigma_{n}}\right|} \frac{\partial}{\partial \cos \theta}\left(\sin ^{2} \theta \frac{\partial}{\partial \cos \theta}\right) \frac{1}{1-\cos \theta\left|\nabla E_{\boldsymbol{P}, \sigma_{n}}\right|} \\
& \quad=2 \pi \tilde{\alpha}\left|\nabla E_{\boldsymbol{P}, \sigma_{n}}\right|^{2} \int_{-1}^{1} d t\left(1-t^{2}\right)\left(\frac{d}{d t} \frac{1}{1-t\left|\nabla E_{\boldsymbol{P}, \sigma_{n}}\right|}\right)^{2} \leq c_{2}, \quad(5.20)
\end{aligned}
$$

where we introduced the variable $t:=\cos \theta$. This bound is uniform in $n$ by Lemma 4.1. Coming back to formula (5.18) and collecting our estimates we obtain

$$
\left\|\psi_{i, n}\right\|_{L_{\mathrm{tr}}^{2}\left(\mathbb{R}^{3} ; \mathbb{C}^{3}\right)}^{2} \leq C \ln \frac{\epsilon_{i}}{\epsilon_{i+1}}\left(\frac{1}{i^{2}}+b_{i}^{2}\right),
$$

where $C$ is independent of $n$. Given the choice of $\varepsilon_{i}, b_{i}, i \in \mathbb{N}$, in Definition 5.1, estimates (5.17) follow. Using this and (5.16), the last statement of the lemma follows by dominated convergence, noting that the $\psi_{i, n}$ are mutually orthogonal.

Using these results we are now ready to show convergence of $T \boldsymbol{v}_{\boldsymbol{P}, \sigma_{n}}$.

Proof of Proposition 4.2 (a). We write

$$
T_{1} \boldsymbol{v}_{\boldsymbol{P}, \sigma_{n}}-T_{1} \boldsymbol{v}_{\boldsymbol{P}}=\sum_{i=1}^{\infty}\left(\psi_{i, n}-\psi_{i}\right)-\sum_{i=n}^{\infty} \psi_{i, n}
$$


In the limit $n \rightarrow \infty$ we apply Lemma 5.3 to the first sum. As for the second term, since

$$
\left\|\sum_{i=n}^{\infty} \psi_{i, n}\right\|_{L_{\mathrm{tr}}^{2}\left(\mathbb{R}^{3} ; \mathbb{C}^{3}\right)}^{2}=\sum_{i=n}^{\infty}\left\|\psi_{i, n}\right\|_{L_{\mathrm{tr}}^{2}\left(\mathbb{R}^{3} ; \mathbb{C}^{3}\right)}^{2}
$$

where $\left\|\psi_{i, n}\right\|_{L_{\mathrm{tr}}^{2}\left(\mathbb{R}^{3} ; \mathbb{C}^{3}\right)}^{2} \leq C_{i}$, see (5.17), it is the reminder term of a convergent series, and therefore vanishes for $n \rightarrow \infty$. This concludes the proof.

\section{Proof of velocity superselection}

The goal of this section is to provide a proof of Proposition 4.4.

Suppose, by contradiction, that $\left[\omega_{\boldsymbol{P}}\right]_{\operatorname{In}(\mathfrak{A})}=\left[\omega_{\boldsymbol{P}^{\prime}}\right]_{\operatorname{In}(\mathfrak{A})}$ for some $\boldsymbol{P} \neq \boldsymbol{P}^{\prime}$ from $\mathcal{S}$. That is, $\omega_{\boldsymbol{P}}=\omega_{\boldsymbol{P}^{\prime}} \circ i_{\boldsymbol{P}, \boldsymbol{P}^{\prime}}$ for some $i_{\boldsymbol{P}, \boldsymbol{P}^{\prime}} \in \operatorname{In}(\mathfrak{A})$. Furthermore, using formula (4.18), we can write

$$
\omega_{\Phi_{\boldsymbol{P}}} \circ \alpha_{\boldsymbol{v}_{\boldsymbol{P}}}=\omega_{\Phi_{\boldsymbol{P}^{\prime}}} \circ \alpha_{\boldsymbol{v}_{\boldsymbol{P}^{\prime}}} \circ i_{\boldsymbol{P}, \boldsymbol{P}^{\prime}},
$$

where, as before, $\omega_{\Phi}(\cdot)=\left\langle\Phi, \pi_{\text {vac }}(\cdot) \Phi\right\rangle$. Again, for any unit vector $\Phi \in \mathcal{F}$ we can find $i_{\Phi} \in \operatorname{In}(\mathfrak{A})$ such that $\omega_{\Phi}=\omega_{\text {vac }} \circ i_{\Phi}$. Hence, we obtain from (6.1) that

$$
\omega_{\mathrm{vac}} \circ \alpha_{\boldsymbol{v}_{\boldsymbol{P}}}=\omega_{\mathrm{vac}} \circ \tilde{i}_{\boldsymbol{P}, \boldsymbol{P}^{\prime}} \circ \alpha_{\boldsymbol{v}_{\boldsymbol{P}^{\prime}}}
$$

for another $\tilde{i}_{\boldsymbol{P}, \boldsymbol{P}^{\prime}} \in \operatorname{In}(\mathfrak{A})$. To disprove this equality, we choose some $\boldsymbol{g} \in \mathcal{L}$, purely imaginary and integrable, set $\boldsymbol{g}_{s}(\boldsymbol{k}):=s^{3 / 2} \boldsymbol{g}(s \boldsymbol{k}), s>0$, and consider the sequence $s \mapsto W\left(\boldsymbol{g}_{s}\right)$. We will evaluate this sequence on the two states appearing in (6.2).

First, using formula (3.4), we get $\alpha_{\boldsymbol{v}_{\boldsymbol{P}}}\left(W\left(\boldsymbol{g}_{s}\right)\right)=e^{-2 i \operatorname{Im}\left\langle\boldsymbol{v}_{\boldsymbol{P}}, \boldsymbol{g}_{s}\right\rangle} W\left(\boldsymbol{g}_{s}\right)$. With the help of (4.11), the fact that $\boldsymbol{g}$ is transverse, and the dominated convergence theorem, we get

$$
\begin{array}{r}
\lim _{s \rightarrow \infty}\left\langle\boldsymbol{v}_{\boldsymbol{P}}, \boldsymbol{g}_{s}\right\rangle=\lim _{s \rightarrow \infty} \tilde{\alpha}^{1 / 2} \int d^{3} k \frac{\chi_{[0, \kappa]}(|\boldsymbol{k}| / s)}{|\boldsymbol{k}|^{3 / 2}} \frac{\nabla E_{\boldsymbol{P}}}{1-\hat{\boldsymbol{k}} \cdot \nabla E_{\boldsymbol{P}}} \cdot \boldsymbol{g}(\boldsymbol{k}) \\
=\tilde{\alpha}^{1 / 2} \int d^{3} k \frac{1}{|\boldsymbol{k}|^{3 / 2}} \frac{\nabla E_{\boldsymbol{P}}}{1-\hat{\boldsymbol{k}} \cdot \nabla E_{\boldsymbol{P}}} \cdot \boldsymbol{g}(\boldsymbol{k}),
\end{array}
$$

and denote the last expression by $\left\langle\boldsymbol{v}_{\boldsymbol{P}}, \boldsymbol{g}_{\infty}\right\rangle$. Since $s \mapsto W\left(\boldsymbol{g}_{s}\right)$ is a central sequence (see Lemma 6.1 below), equality (6.2) and definition (3.6) imply

$$
e^{-2 i \operatorname{Im}\left\langle\boldsymbol{v}_{\boldsymbol{P}}, \boldsymbol{g}_{\infty}\right\rangle}=e^{-2 i \operatorname{Im}\left\langle\boldsymbol{v}_{\boldsymbol{P}^{\prime}}, \boldsymbol{g}_{\infty}\right\rangle} .
$$

Now we want to achieve a contradiction by choosing $\boldsymbol{g}$ so that the above equality fails. For this purpose we first set $\boldsymbol{g}=i \boldsymbol{g}_{1}$, where $\boldsymbol{g}_{1}$ is real. Recalling that $\boldsymbol{v}_{\boldsymbol{P}}$ is real, we use (6.3) to write

$$
\begin{aligned}
\operatorname{Im}\left\langle\boldsymbol{v}_{\boldsymbol{P}}, \boldsymbol{g}_{\infty}\right\rangle-\operatorname{Im}\left\langle\boldsymbol{v}_{\boldsymbol{P}^{\prime}}, \boldsymbol{g}_{\infty}\right\rangle & =\tilde{\alpha}^{1 / 2} \int d^{3} k\left(\frac{\nabla E_{\boldsymbol{P}}}{1-\hat{\boldsymbol{k}} \cdot \nabla E_{\boldsymbol{P}}}-\frac{\nabla E_{\boldsymbol{P}^{\prime}}}{1-\hat{\boldsymbol{k}} \cdot \nabla E_{\boldsymbol{P}^{\prime}}}\right) \cdot \frac{\boldsymbol{g}_{1}(\boldsymbol{k})}{|\boldsymbol{k}|^{3 / 2}} \\
& =\tilde{\alpha}^{1 / 2} \int d^{3} k\left(\frac{\nabla E_{\boldsymbol{P}}-\hat{\boldsymbol{k}}}{1-\hat{\boldsymbol{k}} \cdot \nabla E_{\boldsymbol{P}}}-\frac{\nabla E_{\boldsymbol{P}^{\prime}}-\hat{\boldsymbol{k}}}{1-\hat{\boldsymbol{k}} \cdot \nabla E_{\boldsymbol{P}^{\prime}}}\right) \cdot \frac{\boldsymbol{g}_{1}(\boldsymbol{k})}{|\boldsymbol{k}|^{3 / 2}}
\end{aligned}
$$


where in the second step we used that $\boldsymbol{g}_{1}$ is transverse to take the transverse part of the expression in bracket. We denote the expression in bracket in $(6.5)$ by $F_{\boldsymbol{P}_{, \boldsymbol{P}^{\prime}}}(\hat{\boldsymbol{k}})$ and define $\boldsymbol{g}_{1}$ as follows:

$$
\boldsymbol{g}_{1}(\boldsymbol{k}):=C \frac{F_{\boldsymbol{P}, \boldsymbol{P}^{\prime}}(\hat{\boldsymbol{k}})}{\left|F_{\boldsymbol{P}, \boldsymbol{P}^{\prime}}(\hat{\boldsymbol{k}})\right|^{2}} \chi_{\boldsymbol{P}, \boldsymbol{P}^{\prime}}(\hat{\boldsymbol{k}}) \chi_{[\sigma, \kappa]}(|\boldsymbol{k}|),
$$

where we chose some $C \neq 0,0<\sigma<\kappa$ and $\chi_{\boldsymbol{P}, \boldsymbol{P}^{\prime}}$ is a non-zero, bounded, positive function from $L^{2}\left(S^{2}\right)$ which vanishes near $\operatorname{Span}\left\{\nabla E_{\boldsymbol{P}}, \nabla E_{\boldsymbol{P}^{\prime}}\right\}$. We note that by Lemma 4.1 (a) we have $\nabla E_{\boldsymbol{P}} \neq \nabla E_{\boldsymbol{P}^{\prime}}$ for $\boldsymbol{P} \neq \boldsymbol{P}^{\prime}$.

Now it follows from Lemma 6.2 below that the denominator in (6.6) is non-zero on the support of $\chi_{\boldsymbol{P}, \boldsymbol{P}^{\prime}}$. With this definition, (6.5) gives

$$
\operatorname{Im}\left\langle\boldsymbol{v}_{\boldsymbol{P}}, \boldsymbol{g}_{\infty}\right\rangle-\operatorname{Im}\left\langle\boldsymbol{v}_{\boldsymbol{P}^{\prime}}, \boldsymbol{g}_{\infty}\right\rangle=C \tilde{\alpha}^{1 / 2} \int d^{3} k \chi_{\boldsymbol{P}, \boldsymbol{P}^{\prime}}(\hat{\boldsymbol{k}}) \frac{\chi_{[\sigma, \kappa]}(|\boldsymbol{k}|)}{|\boldsymbol{k}|^{3 / 2}},
$$

which is manifestly non-zero. By varying $C$ we can avoid equality in (6.4) due to the periodicity of the exponential function. This concludes the proof of Proposition 4.4.

Lemma 6.1. For $\boldsymbol{g} \in \mathcal{L} \cap L_{\mathrm{tr}}^{1}\left(\mathbb{R}^{3} ; \mathbb{C}^{3}\right)$ and $s>0$ we set $\boldsymbol{g}_{s}(\boldsymbol{k}):=s^{3 / 2} \boldsymbol{g}($ sk $)$. Then $s \mapsto W\left(\boldsymbol{g}_{s}\right)$ is a central sequence, i.e.,

$$
\lim _{s \rightarrow \infty}\left\|\left[A, W\left(\boldsymbol{g}_{s}\right)\right]\right\|=0 \text { for any } A \in \mathfrak{A} .
$$

Proof. For any given $\boldsymbol{f} \in \mathcal{L}$ we compute the commutator

$$
\left[W(\boldsymbol{f}), W\left(\boldsymbol{g}_{s}\right)\right]=\left(e^{-i \operatorname{Im}\left\langle\boldsymbol{f}, \boldsymbol{g}_{s}\right\rangle}-e^{i \operatorname{Im}\left\langle\boldsymbol{f}, \boldsymbol{g}_{s}\right\rangle}\right) W\left(\boldsymbol{f}+\boldsymbol{g}_{s}\right) .
$$

Now we find $\boldsymbol{f}_{n} \in \mathcal{L}$ which are bounded functions ${ }^{6}$ and such that $\left\|\boldsymbol{f}-\boldsymbol{f}_{n}\right\| \rightarrow 0$ as $n \rightarrow \infty$. The expression in the exponential in the bracket above has the form

$$
\left\langle\boldsymbol{f}, \boldsymbol{g}_{s}\right\rangle=\frac{1}{s^{3 / 2}} \int d^{3} k \overline{\boldsymbol{f}}_{n}(\boldsymbol{k} / s) \cdot \boldsymbol{g}(\boldsymbol{k})+\left\langle\boldsymbol{f}-\boldsymbol{f}_{n}, \boldsymbol{g}_{s}\right\rangle .
$$

Using that $\boldsymbol{g} \in L_{\mathrm{tr}}^{1}\left(\mathbb{R}^{3} ; \mathbb{C}^{3}\right)$ and each $\boldsymbol{f}_{n}$ is bounded, we can apply the dominated convergence theorem to the integral above for any fixed $n$. Exploiting in addition that $\left\|\boldsymbol{g}_{s}\right\|=\|\boldsymbol{g}\|$, we get $\lim _{s \rightarrow \infty}\left\langle\boldsymbol{f}, \boldsymbol{g}_{s}\right\rangle=0$. Consequently, (6.9) gives

$$
\lim _{s \rightarrow \infty}\left\|\left[W(\boldsymbol{f}), W\left(\boldsymbol{g}_{s}\right)\right]\right\|=0 \text { for any } \boldsymbol{f} \in \mathcal{L} .
$$

Since any $A \in \mathfrak{A}$ can be approximated in norm by finite linear combinations of $W(\boldsymbol{f})$, $\boldsymbol{f} \in \mathcal{L}$, the proof is complete.

Lemma 6.2. For any $\boldsymbol{v} \in \mathbb{R}^{3},|\boldsymbol{v}|<1$, consider the function on $S^{2}$

$$
F_{\boldsymbol{v}}(\hat{\boldsymbol{k}}):=\frac{\boldsymbol{v}-\hat{\boldsymbol{k}}}{1-\hat{\boldsymbol{k}} \cdot \boldsymbol{v}} .
$$

Suppose that $\boldsymbol{v}_{1}, \boldsymbol{v}_{2} \in \mathbb{R}^{3}$ are such that $\boldsymbol{v}_{1} \neq \boldsymbol{v}_{2}$. Then, for all $\hat{\boldsymbol{k}} \in S^{2}$ such that $\hat{\boldsymbol{k}} \notin \operatorname{Span}\left\{\boldsymbol{v}_{1}, \boldsymbol{v}_{2}\right\}$, we have

$$
F_{\boldsymbol{v}_{1}}(\hat{\boldsymbol{k}}) \neq F_{\boldsymbol{v}_{2}}(\hat{\boldsymbol{k}}) .
$$

\footnotetext{
${ }^{6}$ i.e., each $\boldsymbol{k} \mapsto\left|\boldsymbol{f}_{n}(\boldsymbol{k})\right|$ is bounded, where $|\cdot|$ means norm in $\mathbb{C}^{3}$.
} 
Proof. Assume $\boldsymbol{v}_{1} \neq \boldsymbol{v}_{2}$ and suppose that

$$
\frac{\boldsymbol{v}_{1}-\hat{\boldsymbol{k}}}{1-\hat{\boldsymbol{k}} \cdot \boldsymbol{v}_{1}}=\frac{\boldsymbol{v}_{2}-\hat{\boldsymbol{k}}}{1-\hat{\boldsymbol{k}} \cdot \boldsymbol{v}_{2}}
$$

Then $\hat{\boldsymbol{k}} \cdot \boldsymbol{v}_{1} \neq \hat{\boldsymbol{k}} \cdot \boldsymbol{v}_{2}$. (If not, then (6.14) implies $\boldsymbol{v}_{1}=\boldsymbol{v}_{2}$ which is a contradiction.)

From (6.14) we have

$$
\hat{\boldsymbol{k}}=\frac{1-\hat{\boldsymbol{k}} \cdot \boldsymbol{v}_{2}}{\hat{\boldsymbol{k}} \cdot \boldsymbol{v}_{1}-\hat{\boldsymbol{k}} \cdot \boldsymbol{v}_{2}} \boldsymbol{v}_{1}-\frac{1-\hat{\boldsymbol{k}} \cdot \boldsymbol{v}_{1}}{\hat{\boldsymbol{k}} \cdot \boldsymbol{v}_{1}-\hat{\boldsymbol{k}} \cdot \boldsymbol{v}_{2}} \boldsymbol{v}_{2} .
$$

This implies $\hat{\boldsymbol{k}} \in \operatorname{Span}\left\{\boldsymbol{v}_{1}, \boldsymbol{v}_{2}\right\}$.

\section{Conclusions}

In this paper we introduced the concept of a relative normalizer for two subgroups $R \subset S$ of a group $G$ given by $N_{G}(R, S):=\left\{g \in G \mid g \cdot S \cdot g^{-1} \subset R\right\}$. We could show that the inhomogeneous symplectic group and the group of automorphisms of the corresponding CCR algebra admit non-trivial relative normalizers. They are given by the infravacuum maps of Kraus, Polley and Reents and the corresponding Bogolubov transformations. Moreover, we studied the impact of such relative normalizers on the superselection theory of this CCR algebra. We gave canonical definitions of the conjugate and second conjugate class of a given superselection sector with respect to a reference 'vacuum' state and a 'background' automorphism. Then we showed that distinct sectors may give rise to coinciding conjugate and second conjugate classes if they are computed with respect to the infravacuum background. This shed a new light on the problem of velocity superselection in non-relativistic QED.

Our findings warrant further investigations of infravacuum representations in QED. One question which is left open is how large the second conjugate classes of states of physical interest are. It is easy to conclude from Lemma 2.4 and Proposition 4.4 that ${\overline{\left[\left[\omega_{\text {vac }}\right]_{\operatorname{In}(\mathfrak{l l})}\right.}}^{\alpha_{T}} \neq{\overline{\overline{\left[\omega_{\text {vac }} \circ \alpha_{T}\right]_{\operatorname{In}(\mathfrak{l l})}}}}^{\alpha_{T}}$, thus in general second conjugate classes are proper subsets of the set of pure states. We showed in Theorem 2.9 (c) that second conjugate classes of physical states contain their usual soft-photon dressing, given by singular coherent representations. However, we do not know if the inclusion in this latter result is an equality or not. As many other examples of soft-photon clouds can be found in the literature (see, e.g., [BD84]) it is not obvious that the equality should hold.

Another interesting direction is a resolution of velocity superselection at the level of states, following up on Theorem 4.6. The non-canonical character of the decomposition (4.23) could probably be overcome by forming suitable equivalence classes of all possible decompositions into the 'undressed electron' and the 'dressing'. However, we believe that there is a more natural approach to this problem which is less dependent on the infravacuum maps $T$ : This is to choose the symplectic space $\mathcal{L}$ in such a way that the algebra $\mathfrak{A}$ has a local structure and consider the restrictions of $\omega_{\boldsymbol{P}}$ to the subalgebra $\mathfrak{A}\left(V_{+}\right) \subset \mathfrak{A}$ of the electromagnetic fields localized in the future 
lightcone $V_{+}$. We remark that such a choice of $\mathcal{L}$ is by no means prevented by the non-relativistic character of the considered model. We conjecture that the (highly reducible) GNS representations of $\omega_{\boldsymbol{P}} \uparrow \mathfrak{A}\left(V_{+}\right)$are unitarily equivalent for different $\boldsymbol{P}$

and that they are closely related to the second conjugate classes ${\overline{\overline{\left.\left[\omega_{\boldsymbol{P}}\right]_{\operatorname{In}(\mathfrak{A})}\right]}}}^{\alpha_{T}}$ considered in the present work. This research direction, which will be pursued elsewhere, aims at a verification in a concrete model of the general ideas from [BR14, AD17].

Yet another important direction is to address the problem of the sharp mass of the electron in non-relativistic QED. For this purpose one needs to transform the model Hamiltonian to a Kraus-Polley-Reents representation in a suitable way. We remark in this context that the formal expression $\alpha_{T^{-1}}\left(H_{\boldsymbol{P}}\right)$ appears to have infraredregularized interaction terms, hinting at a possible presence of ground states. On the other hand, $\alpha_{T^{-1}}$ acts also on $H_{\mathrm{ph}}$ and $P_{\mathrm{ph}}$ which enter in the formula for $H_{\boldsymbol{P}}$. The resulting modification seems in conflict with the existence of ground states, even in the absence of interaction. Thus we believe that a naive application of $\alpha_{T^{-1}}$ as a dressing transformation at time zero will not yield a sharp mass of the electron yet. It may help to express $\alpha_{T^{-1}}$ in terms of asymptotic (incoming and outgoing) fields and define accordingly two infravacuum Hamiltonians. This strategy is consistent with recent works in the setting of algebraic QFT which stress the role of the arrow of time for curing the infrared problems [BR14, AD17].

On a more speculative side, we think the proposal of Hawking, Perry and Strominger [HPS16] concerning the black-hole information paradox should be reconsidered from the infravacuum perspective. If the relevant infrared degrees of freedom of the gravitational field can be blurred by infravacuum-type radiation of arbitrarily low energy, can they really encode information about the history of the black-hole formation? We hope to come back to the above questions in future publications.

\section{A Some auxiliary lemmas about $C^{*}$-algebras}

For the reader's convenience we recall some standard facts from the theory of $C^{*}$ algebras which we use in our paper. We refer to [KR, Chapter 10] for a more extensive discussion.

Lemma A.1. Let $(\pi, \mathcal{H})$ be an irreducible representation of a $C^{*}$-algebra $\mathfrak{A}$ and let $\Psi \in \mathcal{H}$ be a unit vector. Then the GNS representation $(\tilde{\pi}, \tilde{\mathcal{H}}, \tilde{\Omega})$ induced by the state

$$
\omega(A)=\langle\Psi, \pi(A) \Psi\rangle, \quad A \in \mathfrak{A},
$$

is unitarily equivalent to $(\pi, \mathcal{H})$.

Proof. The map $V: \mathcal{H} \rightarrow \tilde{\mathcal{H}}$, given by $V \pi(A) \Psi=\tilde{\pi}(A) \tilde{\Omega}, A \in \mathfrak{A}$, is densely defined by irreducibility of $\pi$ and has a dense range by cyclicity of $\tilde{\Omega}$. By a straightforward computation one checks that $V$ is an isometry and $V \pi(A)=\tilde{\pi}(A) V$ for all $A \in \mathfrak{A}$.

In the following proposition we write $[\omega]:=[\omega]_{\operatorname{In}(\mathfrak{A})}$ for brevity.

Proposition A.2. Let $\omega$ be a pure state on $\mathfrak{A}$ and $(\pi, \mathcal{H}, \Omega)$ its $G N S$ representation. Then $[\omega]$ coincides with the set of all states whose GNS representations are unitarily 
equivalent to $\pi$. Furthermore, $[\omega \circ \gamma]=[\omega]$ iff $\gamma \in \operatorname{Aut}(\mathfrak{A})$ is unitarily implementable in $\pi$.

Proof. Let $\omega_{1} \in[\omega]$ and denote by $\left(\pi_{1}, \mathcal{H}_{1}, \Omega_{1}\right)$ its GNS representation. Since $\omega_{1}=$ $\omega \circ \operatorname{Ad} U$ for some unitary $U \in \mathfrak{A}$, we can write

$$
\begin{aligned}
& \omega(A)=\langle\Omega, \pi(A) \Omega\rangle, \\
& \omega_{1}(A)=\left\langle\Omega_{1}, \pi_{1}(A) \Omega_{1}\right\rangle=\left\langle\Omega, \pi\left(U A U^{*}\right) \Omega\right\rangle=\left\langle\pi\left(U^{*}\right) \Omega, \pi(A) \pi\left(U^{*}\right) \Omega\right\rangle,
\end{aligned}
$$

for $A \in \mathfrak{A}$. Now Lemma A.1 gives unitary equivalence of $\pi$ and $\pi_{1}$.

Conversely, suppose that $\pi$ and $\pi_{1}$ are unitarily equivalent, i.e., $\pi_{1}(A)=V \pi(A) V^{*}$ for all $A \in \mathfrak{A}$ and some unitary $V: \mathcal{H} \rightarrow \mathcal{H}_{1}$. Thus we can write

$$
\omega_{1}(A)=\left\langle\Omega_{1}, V \pi(A) V^{*} \Omega_{1}\right\rangle=\left\langle\Omega, \pi(U) \pi(A) \pi(U)^{*} \Omega\right\rangle=\omega\left(U A U^{*}\right),
$$

where we used the irreducibility of $\pi$ and the resulting existence of a unitary $U \in \mathfrak{A}$ such that $\pi(U)^{*} \Omega=V^{*} \Omega_{1}$. This follows from the Kadison transitivity theorem [KR, Theorem 10.2.1].

As for the last statement, suppose that $\gamma$ is unitarily implementable in $\pi$, that is,

$$
\pi \circ \gamma=\operatorname{Ad} U_{\gamma} \circ \pi
$$

for some unitary $U_{\gamma}$ on $\mathcal{H}$. Thus we can write for any $A \in \mathfrak{A}$

$$
\begin{aligned}
\omega(\gamma(A)) & =\langle\Omega, \pi(\gamma(A)) \Omega\rangle=\left\langle U_{\gamma}^{*} \Omega, \pi(A) U_{\gamma}^{*} \Omega\right\rangle \\
& =\left\langle\pi\left(V_{\gamma}^{*}\right) \Omega, \pi(A) \pi\left(V_{\gamma}^{*}\right) \Omega\right\rangle=\omega\left(V_{\gamma} A V_{\gamma}^{*}\right),
\end{aligned}
$$

where we used again $\left[\mathrm{KR}\right.$, Theorem 10.2.1] to find a unitary $V_{\gamma} \in \mathfrak{A}$ such that $\pi\left(V_{\gamma}^{*}\right) \Omega=U_{\gamma}^{*} \Omega$.

Now suppose that $\omega$ and $\omega \circ \gamma$ are in the same sector, i.e., $\omega=\omega \circ \gamma \circ i$ for some $i \in \operatorname{In}(\mathfrak{A})$. Then $\gamma \circ i$ leaves $\omega$ invariant, hence it is unitarily implementable by the GNS theorem. As any $i \in \operatorname{In}(\mathfrak{A})$ is unitarily implementable, we conclude the proof.

\section{B Vector valued spherical harmonics}

As we did not find a satisfactory reference, in this appendix we summarize the basic properties of the vector valued spherical harmonics from Section 5:

$$
\boldsymbol{Y}_{\ell m \pm}=\frac{1}{\sqrt{\ell(\ell+1)}} \boldsymbol{a}_{ \pm} Y_{\ell m} \quad \text { with } \quad \boldsymbol{a}_{+}=|k| \nabla_{\boldsymbol{k}} \quad \text { and } \quad \boldsymbol{a}_{-}=\hat{\boldsymbol{k}} \times \boldsymbol{a}_{+},
$$

where $Y_{\ell m}$ are the usual spherical harmonics, orthonormal with respect to the measure $d \Omega(\theta, \phi)=\sin \theta d \theta d \phi$. For this purpose we recall that the total angular momentum of a photon is a self-adjoint operator on $L_{\mathrm{tr}}^{2}\left(\mathbb{R}^{3} ; \mathbb{C}^{3}\right)$ given by

$$
\boldsymbol{J}=\boldsymbol{L}+\boldsymbol{S}, \quad \boldsymbol{L}:=-i \boldsymbol{k} \times \nabla_{\boldsymbol{k}}, \quad \boldsymbol{S}=\left(S_{1}, S_{2}, S_{3}\right), \quad\left(S_{k} \boldsymbol{\psi}\right):=i \boldsymbol{e}_{k} \times \boldsymbol{\psi},
$$

where $\boldsymbol{\psi} \in L_{\mathrm{tr}}^{2}\left(\mathbb{R}^{3} ; \mathbb{C}^{3}\right)$ and $\left\{\boldsymbol{e}_{k}\right\}_{k=1,2,3}$ is the canonical basis in $\mathbb{R}^{3}$. The operators $\left\{S_{k}\right\}_{k=1,2,3}$ satisfy the standard angular momentum commutation relations and $\boldsymbol{S}^{2}=2$ holds true (cf. [LL, §58, Problem 2]). 
Proposition B.1. The vector valued spherical harmonics $\boldsymbol{Y}_{\ell m \pm}, \ell \in \mathbb{N},-\ell \leq m \leq \ell$, given by equation (B.1)

(1) satisfy $\boldsymbol{J}^{2} \boldsymbol{Y}_{\ell m \pm}=\ell(\ell+1) \boldsymbol{Y}_{\ell m \pm}$ and $\boldsymbol{J}_{3} \boldsymbol{Y}_{\ell m \pm}=m \boldsymbol{Y}_{\ell m \pm}$;

(2) form (a) an orthonormal and (b) complete basis of $L_{\mathrm{tr}}^{2}\left(S^{2} ; \mathbb{C}^{3}\right)$.

Proof. (1) Following [BLP], we compute on $C^{2}$ functions from $L_{\mathrm{tr}}^{2}\left(S^{2} ; \mathbb{C}^{3}\right)$

$$
\left[L_{i}, a_{ \pm p}\right]=i \epsilon_{i p q} a_{ \pm q},
$$

where $L_{i}$ and $a_{p}$ are the Cartesian components of $\boldsymbol{L}, \boldsymbol{a}$, respectively, and $\epsilon_{i p q}$ is the Levi-Civita symbol. Since $\left(S_{i} \psi\right)_{p}=-i \epsilon_{i p q} \psi_{q}$, equation (B.3) applied to $Y_{\ell m}$ (which are smooth functions [Ho65, Chapter IV]) can be written as

$$
L_{i} a_{ \pm p} Y_{\ell m}-a_{ \pm p} L_{i} Y_{\ell m}=-\left(S_{i} \boldsymbol{a}_{ \pm} Y_{\ell m}\right)_{p}
$$

which implies

$$
\left(J_{i} \boldsymbol{a}_{ \pm} Y_{\ell m}\right)_{p}=L_{i} a_{ \pm p} Y_{\ell m}+\left(S_{i} \boldsymbol{a}_{ \pm} Y_{\ell m}\right)_{p}=a_{ \pm p} L_{i} Y_{\ell m} .
$$

Consequently, for $i=3$ the above equation yields

$$
J_{3}\left(\boldsymbol{a}_{ \pm} Y_{\ell m}\right)=\boldsymbol{a}_{ \pm} L_{3} Y_{\ell m}
$$

Instead, by applying $J_{i}$ twice and summing over the index $i=1,2,3$, we find from (B.5),

$$
\boldsymbol{J}^{2}\left(\boldsymbol{a}_{ \pm} Y_{\ell m}\right)=\boldsymbol{a}_{ \pm} \boldsymbol{L}^{2} Y_{\ell m}
$$

Since the scalar spherical harmonics are eigenfunctions of the operators $\boldsymbol{L}^{2}$ and $L_{3}$ with eigenvalues $\ell(\ell+1)$ and $m$, respectively, we arrive at (1).

(2a) We denote by $\nabla_{t}=\boldsymbol{a}_{+}$the gradient on the sphere. Then the $\boldsymbol{Y}_{\ell m+}$ are orthogonal to the $\boldsymbol{Y}_{\ell^{\prime} m^{\prime}-}$, which follows by Green's identity on $S^{2}$. Similarly for $\boldsymbol{Y}_{\ell m+}$,

$$
\begin{aligned}
\frac{1}{\ell(\ell+1)} \int_{S^{2}} \nabla_{t} \overline{Y_{\ell m}(\hat{\boldsymbol{k}})} & \cdot \nabla_{t} Y_{\ell^{\prime} m^{\prime}}(\hat{\boldsymbol{k}}) d \Omega \\
& =-\frac{1}{\ell(\ell+1)} \int_{S^{2}} \Delta_{t} \overline{Y_{\ell m}(\hat{\boldsymbol{k}})} Y_{\ell^{\prime} m^{\prime}}(\hat{\boldsymbol{k}}) d \Omega=\delta_{\ell \ell^{\prime}} \delta_{m m^{\prime}},
\end{aligned}
$$

since $Y_{\ell m}$ are orthonormal eigenfunctions of $\Delta_{t}$ with eigenvalues $-\ell(\ell+1)$. As for $Y_{\ell m-}$, we have

$$
\begin{aligned}
& \int_{S^{2}}\left(\hat{\boldsymbol{k}} \times \overline{\boldsymbol{Y}_{\ell m+}(\hat{\boldsymbol{k}})}\right) \cdot\left(\hat{\boldsymbol{k}} \times \boldsymbol{Y}_{\ell^{\prime} m^{\prime}+}(\hat{\boldsymbol{k}})\right) d \Omega= \\
& \int_{S^{2}}(\hat{\boldsymbol{k}} \cdot \hat{\boldsymbol{k}})\left(\overline{\boldsymbol{Y}_{\ell m+}(\hat{\boldsymbol{k}})} \cdot \boldsymbol{Y}_{\ell^{\prime} m^{\prime}+}(\hat{\boldsymbol{k}})\right)-\left(\hat{\boldsymbol{k}} \cdot \boldsymbol{Y}_{\ell^{\prime} m^{\prime}+}(\hat{\boldsymbol{k}})\right)\left(\overline{\boldsymbol{Y}_{\ell m+}(\hat{\boldsymbol{k}})} \cdot \hat{\boldsymbol{k}}\right) d \Omega=\delta_{\ell \ell^{\prime}} \delta_{m m^{\prime}},
\end{aligned}
$$

using the above result for $Y_{\ell m+}$ and the fact that these are orthogonal to $\hat{k}$. 
(2b) Completeness is a consequence of [Wi57, Theorem 3.4], which states that given a field of tangent vectors $\boldsymbol{\alpha}$ in the class $C^{3}$ on the unit sphere $S^{2}$, there exist unique functions $F$ and $G$ of class $C^{2}$ on $S^{2}$ such that

$$
\int_{S^{2}} F d \Omega=\int_{S^{2}} G d \Omega=0
$$

and

$$
\alpha=\nabla_{t} F+\hat{k} \times \nabla_{t} G
$$

We consider an arbitrary smooth field of tangent vectors $\boldsymbol{\alpha}$ on $S^{2}$. As this is more restrictive than the hypothesis in Wilcox's theorem above, we can apply this theorem to $\alpha$, yielding functions $F$ and $G$ of class $C^{2}$ on $S^{2}$ with the properties (B.10) and (B.11) above.

Let us now decompose $F, G$ into sums which converge in $L^{2}\left(S^{2}\right)$ :

$$
F=\sum_{\ell, m} c_{\ell m} Y_{\ell m}, \quad G=\sum_{\ell, m} d_{\ell m} Y_{\ell m}
$$

and substitute them to equation (B.11). In order to exchange the sums with the action of $\nabla_{t},\left(\hat{\boldsymbol{k}} \times \nabla_{t}\right)$, we do the following computation for any $\boldsymbol{\alpha}_{1}$ in the domain of the adjoint maps $\nabla_{t}^{*},\left(\hat{\boldsymbol{k}} \times \nabla_{t}\right)^{*}$. (For example, we can choose $\boldsymbol{\alpha}_{1}$ smooth. As we indicate below, such vector fields form a dense subspace in $L_{\mathrm{tr}}^{2}\left(S^{2} ; \mathbb{C}^{3}\right)$ ).

$$
\begin{aligned}
\left\langle\boldsymbol{\alpha}_{1}, \boldsymbol{\alpha}\right\rangle & =\left\langle\boldsymbol{\alpha}_{1}, \nabla_{t} F+\hat{\boldsymbol{k}} \times \nabla_{t} G\right\rangle \\
& =\left\langle\left(\nabla_{t}\right)^{*} \boldsymbol{\alpha}_{1}, F\right\rangle+\left\langle\left(\hat{\boldsymbol{k}} \times \nabla_{t}\right)^{*} \boldsymbol{\alpha}_{1}, G\right\rangle \\
& =\sum_{\ell, m} c_{\ell, m}\left\langle\left(\nabla_{t}\right)^{*} \boldsymbol{\alpha}_{1}, Y_{\ell m}\right\rangle+\sum_{\ell, m} d_{\ell, m}\left\langle\left(\hat{\boldsymbol{k}} \times \nabla_{t}\right)^{*} \boldsymbol{\alpha}_{1}, Y_{\ell m}\right\rangle \\
& =\sum_{\ell, m} c_{\ell, m}\left\langle\boldsymbol{\alpha}_{1}, \nabla_{t} Y_{\ell m}\right\rangle+\sum_{\ell, m} d_{\ell, m}\left\langle\boldsymbol{\alpha}_{1},\left(\hat{\boldsymbol{k}} \times \nabla_{t}\right) Y_{\ell m}\right\rangle \\
& =\sum_{\ell, m} c_{\ell, m} \sqrt{\ell(\ell+1)}\left\langle\boldsymbol{\alpha}_{1}, \boldsymbol{Y}_{\ell m+}\right\rangle+\sum_{\ell, m} d_{\ell, m} \sqrt{\ell(\ell+1)}\left\langle\boldsymbol{\alpha}_{1}, \boldsymbol{Y}_{\ell m-}\right\rangle .
\end{aligned}
$$

To proceed, we need to show that

$$
\sum_{\ell, m}\left|c_{\ell, m}\right|^{2} \ell(\ell+1)<\infty, \quad \sum_{\ell, m}\left|d_{\ell, m}\right|^{2} \ell(\ell+1)<\infty .
$$

To this end, we compute using formula (B.11)

$$
\begin{aligned}
\left\langle\boldsymbol{Y}_{\ell m+}, \boldsymbol{\alpha}\right\rangle & =\frac{1}{\sqrt{\ell(\ell+1)}}\left\langle\nabla_{t} Y_{\ell m}, \boldsymbol{\alpha}\right\rangle=\frac{1}{\sqrt{\ell(\ell+1)}}\left\langle\nabla_{t} Y_{\ell m}, \nabla_{t} F\right\rangle \\
& =-\frac{1}{\sqrt{\ell(\ell+1)}}\left\langle\Delta_{t} Y_{\ell m}, F\right\rangle=-\sqrt{\ell(\ell+1)}\left\langle Y_{\ell m}, F\right\rangle .
\end{aligned}
$$

Since $c_{\ell, m}=\left\langle Y_{\ell m}, F\right\rangle$ and $Y_{\ell m+}$ form an orthonormal system, the first bound in (B.14) follows. The second bound is proven analogously. 
Given (B.14), computation (B.13) gives

$$
\left\langle\boldsymbol{\alpha}_{1}, \boldsymbol{\alpha}-\sum_{\ell m \pm} \tilde{c}_{\ell m \pm} \boldsymbol{Y}_{\ell m \pm}\right\rangle=0
$$

for some square-summable coefficients $\tilde{c}_{\ell m \pm}$. By taking supremum over all $\boldsymbol{\alpha}_{1}$ in the dense domain specified above, subject to $\left\|\boldsymbol{\alpha}_{1}\right\| \leq 1$, we get

$$
\left\|\boldsymbol{\alpha}-\sum_{\ell m \pm} \tilde{c}_{\ell m \pm} \boldsymbol{Y}_{\ell m \pm}\right\|=0
$$

which implies that any smooth $\boldsymbol{\alpha}$ is in the closed subspace spanned by $\boldsymbol{Y}_{\ell m \pm}$.

Hence it only remains to be shown that the smooth vector fields on the unit sphere are $L^{2}$-dense in the space of all $L^{2}$ vector fields. For this, we consider a generic $L^{2}$ vector field $\boldsymbol{\beta}$ and we split it into a sum $\boldsymbol{\beta}=\boldsymbol{\beta}_{n}+\boldsymbol{\beta}_{s}$, where $\boldsymbol{\beta}_{n, s}$ have support in the north and south hemisphere, respectively. Now stereographic projections map each hemisphere to a circle in $\mathbb{R}^{2}$, and the transformed $\boldsymbol{\beta}_{n, s}$ can be approximated by smooth vector fields on a slightly larger circle. Applying the inverse transformation yields the result.

\section{References}

[AD17] S. Alazzawi and W. Dybalski. Compton scattering in the Buchholz-Roberts framework of relativistic QED. Lett. Math. Phys. 107, (2017) 81-106.

[BFS98] V. Bach, J. Fröhlich and I. M. Sigal. Quantum electrodynamics of confined nonrelativistic particles. Adv. Math. 137, (1998) 299-395.

[BCFFS13] V. Bach, T. Chen, J. Fröhlich, and I. M. Sigal. Effective Dynamics of an electron coupled to an external potential in non-relativistic QED. Ann. Henri Poincaré 6, (2013) 1573-1597.

[BLP] V. B. Berestetskii, E. M. Lifshitz and L. P. Pitaevskii. Relativistic Quantum Theory. Volume 4 of Course of Theoretical Physics, Part 1. Pergamon Press, 1971.

[BR] O. Bratteli and D. W. Robinson. Operator algebras and quantum statistical mechanics I. Springer, 1987.

[Bu82] D. Buchholz. The physical state space of quantum electrodynamics. Commun. Math. Phys. 85, (1982) 49-71.

[BD84] D. Buchholz and S. Doplicher. Exotic infrared representations of interacting systems Annales de l'Institut Henri Poincare. Section A, Physique Theorique. 40, (1984) 175-184.

[Bu86] D. Buchholz. Gauss' law and the infraparticle problem. Phys. Lett. B 174, (1986) 331-334. 
[BR14] D. Buchholz and J.E. Roberts. New light on infrared problems: sectors, statistics, symmetries and spectrum. Commun. Math. Phys. 330, (2014) 935-972.

[Ch08] T. Chen. Infrared renormalization in non-relativistic QED and scaling criticality. J. Funct. Anal. 254, (2008) 2555-2647.

[CF07] T. Chen and J. Fröhlich. Coherent infrared representations in non-relativistic QED. Spectral Theory and Mathematical Physics: A Festschrift in Honor of Barry Simon's 60th Birthday, Vol. I, Proc. Symp. Pure Math., AMS (2007).

[CFP07] T. Chen, J. Fröhlich and A. Pizzo. Infraparticle scattering states in nonrelativistic QED: I. The Bloch-Nordsieck paradigm. Commun. Math. Phys. 294, (2010) 761-825.

[CFP09] T. Chen, J. Fröhlich and A. Pizzo. Infraparticle scattering states in nonrelativistic quantum electrodynamics. II. Mass shell properties. Journal of Mathematical Physics 50, (2009) 012103.

[DG04] J. Dereziński and C. Gérard. Scattering theory of infrared divergent PauliFierz Hamiltonians. Ann. Henri Poincaré 5, (2004) 523-577.

[DG] J. Dereziński and C. Gérard. Mathematics of quantization and quantum fields. Cambridge University Press, 2013.

[DT12] W. Dybalski and Y. Tanimoto. Infraparticles with superselected direction of motion in two-dimensional conformal field theory. Commun. Math. Phys. 311, (2012) 457-490.

[DP13] W. Dybalski and A. Pizzo. Coulomb scattering in the massless Nelson model II. Regularity of ground states. Rev. Math. Phys. 31, (2019) 1950010.

[DP18] W. Dybalski and A. Pizzo. Coulomb scattering in the massless Nelson model III. Ground state wave functions and non-commutative recurrence relations. Ann. Henri Poincaré 19, (2018) 463-514.

[Fr73] J. Fröhlich. On the infrared problem in a model of scalar electrons and massless, scalar bosons. Ann. Inst. H. Poincaré Sect. A (N.S.) 19, (1973) 1-103.

[Fr74] J. Fröhlich. Existence of dressed one electron states in a class of persistent models. Fortschr. Phys. 22, (1974) 159-198.

[FP10] J. Fröhlich and A. Pizzo. Renormalized electron mass in non-relativistic QED. Commun. Math. Phys. 294, (2010) 439-470.

[Ha] R. Haag. Local Quantum Physics. Second edition. Springer-Verlag, Berlin, 1996.

[HH08] D. Hasler and I. Herbst. Absence of ground states for a class of translation invariant models of non-relativistic QED. Commun. Math. Phys. 279, (2008) 769-787. 
[HPS16] S. W. Hawking, M. J. Perry and A. Strominger. Soft hair on black holes. Phys. Rev. Lett. 116, (2016) 231301.

[HiO0] F. Hiroshima. Essential self-adjointness of translation-invariant quantum field models for arbitrary coupling constant. Commun. Math. Phys. 211, (2000) 585-613.

[Ho65] E. W. Hobson. The theory of spherical and ellipsoidal harmonics. Chelsea Publishing Company, 1965.

[KR] R.V. Kadison and J.R. Ringrose. Fundamentals of the theory of operator algebras: Advanced theory. Academic Press, 1986.

[KM14] M. Könenberg, O. Matte. The mass shell in the semi-relativistic Pauli-Fierz model. Ann. Henri Poincaré 15, (2014) 863-915.

[KPR77] K. Kraus, L. Polley and G. Reents. Models for infrared dynamics. I. Classical currents. Ann. Inst. H. Poincaré 26, (1977) 109-162.

[Kr82] K. Kraus. Aspects of the infrared problem in quantum electrodynamics. Found. Phys. 13, (1983) 701-713.

[Ku] W. Kunhardt. On infravacua and the superselection structure of theories with massless particles PhD Thesis, 2001. Available as arXiv:math-ph/0109001.

[Ku98] W. Kunhardt. On infravacua and the localization of sectors. J. Math. Phys. 39, (1998) 6353.

[Ku98.1] W. Kunhardt. On infravacua and superselection theory. J. Math. Phys. 39, (1998) 3589.

[LL] L. D. Landau and E. M. Lifshitz. Quantum Mechanics. Volume 3 of Course of Theoretical Physics. Pergamon Press, 1965.

[LL04] E. H. Lieb and M. Loss. A note on polarization vectors in Quantum Electrodynamics. Commun. Math. Phys. 252, (2004) 477-483.

[LMS07] M. Loss, T. Miyao and H. Spohn. Lowest energy states in nonrelativistic QED: Atoms and ions in motion. J. Funct. Anal. 243, (2007) 353-393.

[Pe89] D. Petz. Leuven notes in Mathematical and Theoretical Physics. Volume 2: An invitation to the algebra of canonical commutation relations. Leuven University Press, 1989.

[Pi03] A. Pizzo. One-particle (improper) states in Nelson's massless model. Ann. Henri Poincaré 4, (2003) 439-486.

[Pi05] A. Pizzo. Scattering of an infraparticle: the one particle sector in Nelson's massless models. Ann. Henri Poincaré 6, (2005) 553-606. 
[Re74] G. Reents. Scattering of photons by an external current. J. Math. Phys. 15, (1974) 31-34.

[Ro70] G. Roepstorff. Coherent photon states and spectral condition. Commun. Math. Phys. 19, (1970) 301-314.

[Ru78] S.N.M. Ruijsenaars. On Bogolubov transformations. II. The general case. Annals of Physics 116, (1978) 105-134.

[Sch16] W. Schmidt. Lie groups and Lie algebras. Lecture notes by Tony Feng, 2012. (http://web.stanford.edu/ tonyfeng/222.pdf)

[Sp] H. Spohn. Dynamics of charged particles and their radiation field. Cambridge University Press, 2004.

[We] S. Weinberg. The quantum theory of fields. Volume 1: Foundations. Cambridge University Press, 2005.

[Wi57] C. H. Wilcox. Debye potentials. Journal of Mathematics and Mechanics 6, (1957) 167-201. 